\title{
Restimulation-induced apoptosis of $T$ cells is impaired in patients with X-linked lymphoproliferative disease caused by SAP deficiency
}

\author{
Andrew L. Snow, ${ }^{1}$ Rebecca A. Marsh, ${ }^{2}$ Scott M. Krummey, ${ }^{1}$ Philip Roehrs, ${ }^{2}$
}

Lisa R. Young, ${ }^{3}$ Kejian Zhang, ${ }^{4}$ Jack van Hoff, ${ }^{5}$ Deepali Dhar, ${ }^{1}$ Kim E. Nichols, ${ }^{6}$

Alexandra H. Filipovich, ${ }^{2}$ Helen C. Su, ${ }^{7}$ Jack J. Bleesing, ${ }^{2}$ and Michael J. Lenardo'

1'Laboratory of Immunology, National Institute of Allergy and Infectious Diseases, NIH, Bethesda, Maryland, USA.

${ }^{2}$ Division of Bone Marrow Transplantation/Immune Deficiency, ${ }^{3}$ Division of Pulmonary Medicine, and ${ }^{4}$ Division of Human Genetics, Cincinnati Children's Hospital Medical Center, Cincinnati, Ohio, USA. ${ }^{5}$ Department of Hematology/Oncology, Dartmouth-Hitchcock Medical Center, Lebanon, New Hampshire, USA. ${ }^{6}$ Division of Oncology, Children's Hospital of Philadelphia, Philadelphia, Pennsylvania, USA. ${ }^{2}$ Laboratory of Host Defenses, National Institute of Allergy and Infectious Diseases, NIH, Bethesda, Maryland, USA.

\begin{abstract}
$\mathrm{X}$-linked lymphoproliferative disease (XLP) is a rare congenital immunodeficiency that leads to an extreme, usually fatal increase in the number of lymphocytes upon infection with EBV. It is most commonly defined molecularly by loss of expression of SLAM-associated protein (SAP). Despite this, there is little understanding of how SAP deficiency causes lymphocytosis following EBV infection. Here we show that $T$ cells from individuals with XLP are specifically resistant to apoptosis mediated by TCR restimulation, a process that normally constrains $T$ cell expansion during immune responses. Expression of SAP and the SLAM family receptor NK, T, and B cell antigen (NTB-A) were required for TCR-induced upregulation of key pro-apoptotic molecules and subsequent apoptosis. Further, SAP/NTB-A signaling augmented the strength of the proximal TCR signal to achieve the threshold required for restimulation-induced cell death (RICD). Strikingly, TCR ligation in activated T cells triggered increased recruitment of SAP to NTB-A, dissociation of the phosphatase SHP-1, and colocalization of NTB-A with CD3 aggregates. In contrast, NTB-A and SHP-1 contributed to RICD resistance in XLP T cells. Our results reveal what we believe to be novel roles for NTB-A and SAP in regulating $T$ cell homeostasis through apoptosis and provide mechanistic insight into the pathogenesis of lymphoproliferative disease in XLP.
\end{abstract}

\section{Introduction}

Effective adaptive immunity relies upon the activation and robust clonal expansion of responding $\mathrm{T}$ lymphocytes that orchestrate the elimination of infectious pathogens. However, it is critical to constrain the pool of effector $T$ cells that may, if left unchecked, inflict damage on host tissues. Programmed cell death via apoptosis plays an integral role in expunging effector $\mathrm{T}$ cells at 2 distinct phases of the immune response (1). As foreign antigens are successfully eliminated and $\mathrm{T}$ cell growth cytokines such as IL-2 dissipate, an intrinsic pathway of apoptosis triggered by cytokine withdrawal causes a major contraction phase of the $\mathrm{T}$ cell response, culling all but the small fraction of $T$ cells that later constitute immunological memory. At the peak of the immune response, when antigen and IL- 2 are still abundant, restimulation through the TCR also deletes effector T cells. This autoregulatory program of restimulation-induced cell death (RICD) constitutes a built-in negative feedback mechanism to preclude overt lymphoproliferation and immunopathology and maintain homeostasis (1). We and others have shown that RICD is executed through the action of multiple biochemical pathways, including both death receptor signals (e.g., FAS-FASL) as well as pro-apoptotic Bcl-2 family proteins targeting the mitochondria (e.g., BIM) $(2,3)$. Genetic defects that impair the

Conflict of interest: The authors have declared that no conflict of interest exists. Citation for this article: J. Clin. Invest. 119:2976-2989 (2009). doi:10.1172/JCI39518. function of these main molecular mediators can result in uncontrolled lymphoproliferation, autoimmunity, and lymphomagenesis in both mice and humans $(4,5)$.

$\mathrm{X}$-linked lymphoproliferative disease (XLP) is a rare congenital immunodeficiency originally described by David Purtilo and colleagues in 1975 (6). Its prevalence, estimated at 2-3 per $10^{6}$ males, may be underreported due to failure to recognize the disorder prior to EBV infection $(7,8)$. XLP is usually diagnosed in childhood or adolescence upon presentation of EBV-associated fulminant infectious mononucleosis (FIM)/hemophagocytic lymphohistiocytosis (HLH) (in $\sim 60 \%$ of cases), lymphoproliferative disease (LPD) including lymphoma, and/or hypo-/dysgammaglobulinemia (9). The massive expansion of reactive $\mathrm{T}$ cells as well as $\mathrm{EBV}^{+} \mathrm{B}$ cells that accompanies EBV-associated FIM is fatal in over $90 \%$ of patients that progress to that stage, underscoring the severity of the disease (10). XLP commonly results from deficiency of SLAM-associated protein (SAP), also designated XLP1, caused by mutations in the SH2D1A gene on chromosome Xq24-25 (11-13). SAP is an Src homology 2 (SH2) domain adapter protein involved in the intracellular signaling of the signaling lymphocyte activation molecule (SLAM) family of immunomodulatory receptors. Loss of SAP causes intrinsic defects in lymphocyte function including cytokine production, cytotoxicity, $\mathrm{T}$ cell-dependent humoral immune responses, and NKT cell development $(14,15)$. However, none of these defects 
adequately explain the rapid, lethal phenotype associated with FIM in affected XLP children. Many of these defects are recapitulated in SAP-deficient mice (16-19). Analogous to XLP, severe T cell expansion with associated mortality occurs in SAP-knockout mice challenged with murine $\gamma$ herpesvirus- 68 or chronic lymphocytic choriomeningitis virus $(17,19,20)$. This connection between SAP deficiency and massive $\mathrm{T}$ cell lymphocytosis, and how it might relate to the particular susceptibility to lethal EBVassociated FIM, is not well understood.

Given that LPD is a characteristic feature of congenital disorders of lymphocyte apoptosis, we conjectured that altered lymphocyte homeostasis may also contribute to XLP-related LPD. Chen et al. recently observed a survival advantage for SAP-knockout murine $\mathrm{CD}^{+} \mathrm{T}$ cells 48 hours after primary stimulation, which they characterized as a defect in activation-induced cell death (AICD) (21). As Strasser and colleagues recently pointed out, the term "AICD" is broadly used to refer to any form of lymphocyte death after antigen stimulation and does not distinguish between the possible mechanisms of lymphocyte apoptosis described above (22). We therefore wanted to explicitly test the mechanism of apoptosis induced by TCR restimulation of pre-activated T cells, herein referred to as "RICD" $(22,23)$. In contrast to the p73-mediated death of freshly activated $\mathrm{T}$ cells highlighted by Chen and colleagues, susceptibility to RICD for pre-activated effector T cells requires prolonged exposure to IL-2, upregulation of the Fas death receptor, and most importantly, a strong second stimulation through the TCR upon re-encountering antigen $(1,24)$. The role of SAP and SLAM receptor signaling in this immunoregulatory process has not been explored previously.

We carried out a molecular investigation of several EBV-naive XLP patients who presented with various LPD phenotypes including B cell lymphoma, interstitial lung disease, and macrophage activation syndrome. Strikingly, we found that activated T cells from these patients were specifically and substantially less sensitive to RICD. This phenotype was recapitulated by silencing the expression of either SAP or the single SLAM family receptor NK, $\mathrm{T}$, and $\mathrm{B}$ cell antigen (NTB-A) in normal donor T cells, indicating that both molecules are necessary for optimal TCR-induced apoptosis. TCR restimulation triggered greater recruitment of SAP to NTB-A, concomitant with dissociation of the protein phosphatase SHP-1 and colocalization of NTB-A with CD3 aggregates. This biochemical process was perturbed in XLP T cells, in which NTB-A and SHP-1 acted to decrease RICD sensitivity in the absence of SAP. Furthermore, we show that SAP and NTB-A function to augment TCR-induced signal strength for robust induction of downstream target genes, including the pro-apoptotic mediators FASL and BIM. Our results suggest that XLP patients are inherently susceptible to the development of antigen-induced LPD as a result of compromised RICD, shedding new light on the predisposition of XLP patients to EBV-associated FIM.

\section{Results}

$T$ cells from patients with XLP are resistant to RICD. We initially encountered $4 \mathrm{EBV}$-seronegative patients whose atypical clinical manifestations suggested a diagnosis of XLP. These patients presented with gastrointestinal tract B cell lymphoma (patient 1 [Pt1], Pt2, Pt4), dysgammaglobulinemia (Pt3, Pt4), and LPD associated with HLH-like macrophage activation syndrome $(\mathrm{Pt} 2)$ or $\mathrm{T}$ cell-driven interstitial pneumonitis (Pt3) (see supplemental material for detailed case presentations; available online with this article; doi:10.1172/JCI39518DS1). Pt3 and Pt4 were brothers. Pt1 received a bone marrow transplant before this research began and therefore was not included in subsequent analyses.

SH2D1A genomic DNA sequencing detected a gross deletion spanning exons 2-4 in Pt1, exons 1-4 in Pt2, and an insertional mutation in Pt3 and Pt4 (195_196insT), resulting in a frame shift and predicted premature stop codon (A66fsX67). Both mutations resulted in a SAP-null phenotype, based on complete absence of SAP protein in immunoblots of patient $\mathrm{T}$ cell lysates (Figure 1A). Sequence analysis of carrier 1 (Cr1, the mother of Pt3 and Pt4) confirmed her obligate carrier state, although total SAP protein expression was comparable to control lysates. Intracellular flow cytometry also failed to detect SAP expression in these patients (Supplemental Figure 1). Clinical immunodiagnostic phenotyping of these patients revealed various hallmarks of XLP disease related to SAP deficiency, including low numbers of $\mathrm{CD} 27^{+}$memory $\mathrm{B}$ cells, poor Ab responses to vaccination, impaired CTL/NK cell cytolytic activity, and absence of NKT cells. On the other hand, we also detected elevated serum sIL-2R $\alpha$ in Pt 3 and increased percentages of perforin/granzyme B-expressing $\mathrm{CD}^{+} \mathrm{T}$ cells in all 3 patients, suggesting an underlying predisposition to immune overactivation. Routine $\mathrm{T}$ cell proliferation assays showed normal or enhanced responses to mitogens or recall antigens. These data are summarized in Supplemental Table 1.

Given the evidence of immune hyperactivation and LPD noted in these patients, we hypothesized that an inherent defect in apoptosis of activated T cells might contribute to disease pathogenesis in XLP. To screen for such defects, activated $T$ cells derived from the patients were tested for sensitivity to apoptosis triggered by various extrinsic and intrinsic apoptosis stimuli. Compared with normal controls and $\mathrm{Cr} 1$, T cells from all 3 patients exhibited significant resistance to apoptosis induced by TCR restimulation (Figure 1B). Resistance to RICD was noted whether restimulation was induced by OKT3 mAb or through $\mathrm{T}$ cell mitogens concanavalin A or phytohemagglutinin (Supplemental Figure 2A). However, XLP patient T cells remained fully sensitive to direct Fas-mediated death as well as intrinsic, mitochondria-dependent apoptosis pathways induced by protein kinase inhibition (staurosporine), DNA damage (UV irradiation), or cytokine deprivation (IL-2 withdrawal) (Figure 1B). TCR restimulation failed to trigger significant phosphatidylserine exposure (Figure $1 \mathrm{C}$ and Supplemental Figure 2B), mitochondrial depolarization, or caspase-3 activation (Figure 1D and Supplemental Figure 2, C and D) in XLP T cells, suggesting that RICD signals were blocked from the outset. These findings were confirmed in activated $T$ cells from an EBV-seropositive patient (Pt5) as well as 3 additional EBVnaive patients (Pt6-Pt8; see Supplemental Figure 3). Furthermore, we found that both $\mathrm{CD}^{+}$and $\mathrm{CD} 8^{+} \mathrm{T}$ cells from XLP patients were resistant to RICD, regardless of whether these subsets were initially activated before or after sorting from bulk PBLs (Figure 1E).

Competence for RICD and Fas-induced apoptosis in effector T cells is contingent on robust activation following primary TCR engagement. We therefore tested whether resistance to RICD simply reflected poor initial $\mathrm{T}$ cell activation. However, all patients and $\mathrm{Cr} 1$ exhibited robust $\mathrm{T}$ cell blast formation and CD25 expression in response to anti-CD3/CD28 stimulation of unfractionated PBLs, similar to normal controls (Supplemental Figure 4A). CD69 upregulation and proliferation of CFSE-labeled T cells from Pt2 and $\mathrm{Pt} 4$ was also comparable to controls regardless of the stimulus used (Supplemental Figure 4B). Only purified XLP CD4 ${ }^{+} \mathrm{T}$ cells showed some diminished CD25 upregulation compared with 


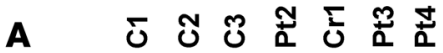

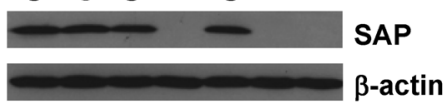

B
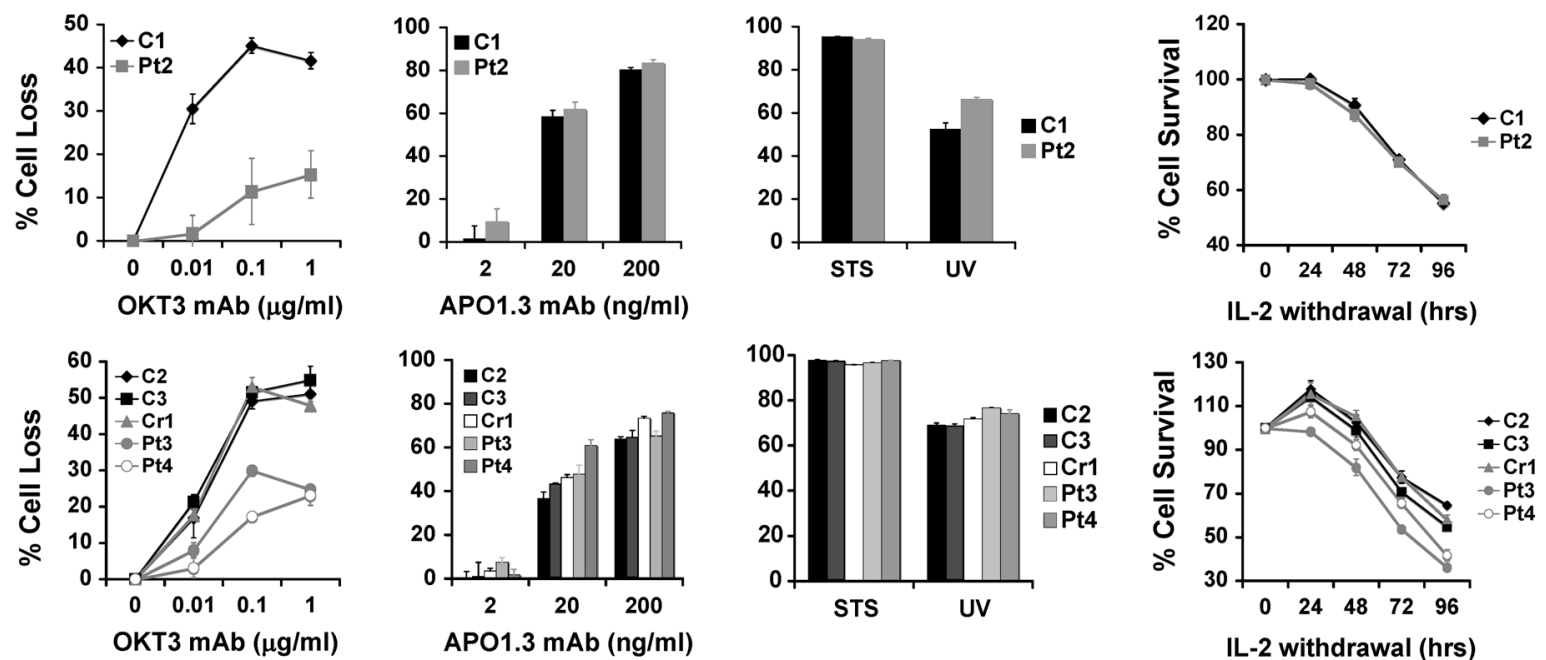

C

OKT3 (hr):

0 8

NC
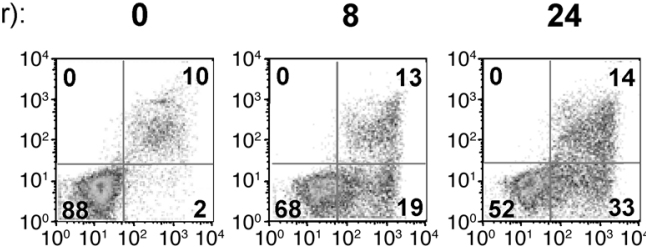

XLP Pt4
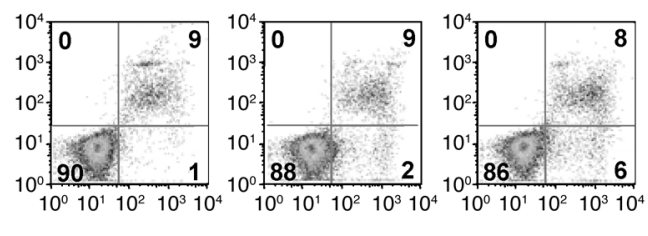

Annexin V

E

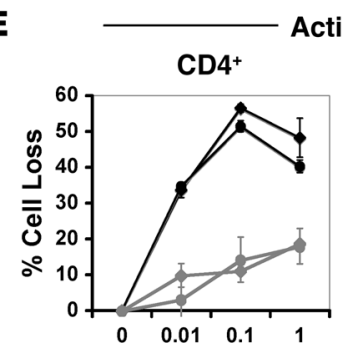

OKT3 mAb $(\mu \mathrm{g} / \mathrm{ml})$
Active PBLs

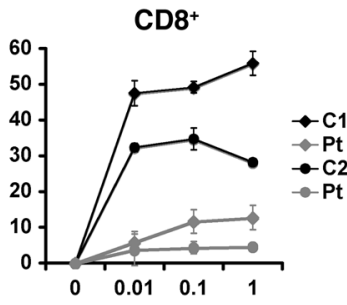

OKT3 mAb $(\mu \mathrm{g} / \mathrm{ml})$
D

IL-2 withdrawal (hrs)

XLP Pt4

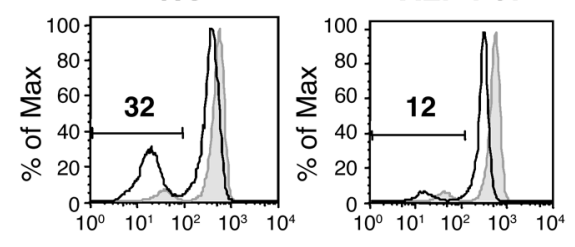

DiOC

$0 \mathrm{hr}$
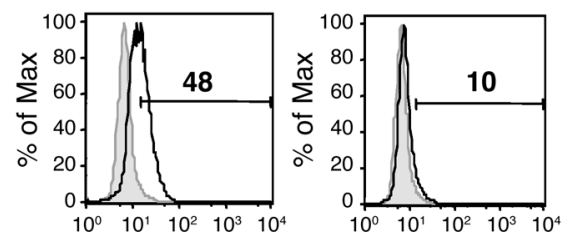

Active Casp 3

Naive PBMCs

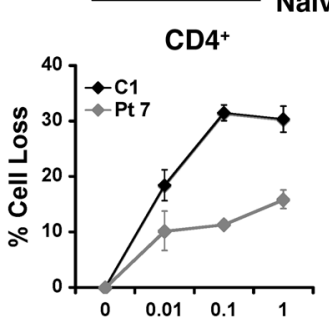

OKT3 mAb ( $\mu \mathrm{g} / \mathrm{ml})$

Figure 1

XLP patient T cells deficient for SAP expression are resistant to RICD. (A) Lysates prepared from activated PBLs were separated by SDS-PAGE and immunoblotted with SAP Ab. $\beta$-Actin served as a loading control. (B) Activated T cells were tested for sensitivity to apoptotic stimuli, including TCR restimulation using anti-CD3 (OKT3) mAb, agonistic anti-Fas APO1.3 mAb, and staurosporine (STS) and UV irradiation, or IL-2 withdrawal. Patient cells (gray) were studied in parallel with normal controls (black; C1-C3) isolated and activated on the same day. Cell loss was measured 24 hours after treatment with propidium iodide (PI) staining and flow cytometry. (C) Activated T cells cultured as described in B were restimulated with $200 \mathrm{ng} / \mathrm{ml}$ OKT3 mAb for 8 and 24 hours or left untreated $(0 \mathrm{hr})$, then stained with Annexin V-FITC and PI. The numbers in each quadrant indicate the percentage of cells in that quadrant. NC, normal control. (D) Activated T cells were restimulated as above for 24 hours or left untreated, then stained with $\mathrm{DiOC}_{6}$ to measure mitochondrial membrane potential (top) or with an Ab for detection of intracellular activated caspase-3 (bottom). The numbers indicate the percentage of cells with permeabilized mitochondria (DiOC6 ${ }^{10}$ ) or active caspase-3 after restimulation. (E) $\mathrm{CD}^{+}$and $\mathrm{CD} 8^{+} \mathrm{T}$ cells were purified from activated PBLs (left) or naive PBMCs (right) and then activated. RICD sensitivity was assessed as described in $\mathbf{B}$. 
A

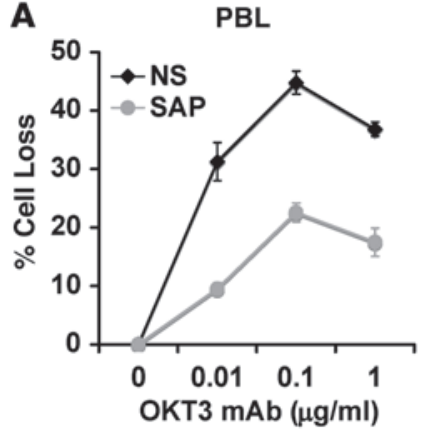

B OKT3 (hr):
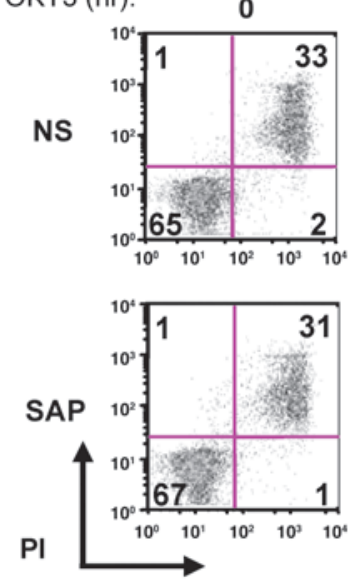

Annexin V
$\mathrm{CD}^{+}$

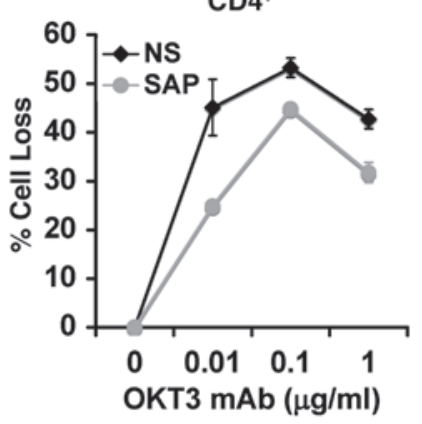

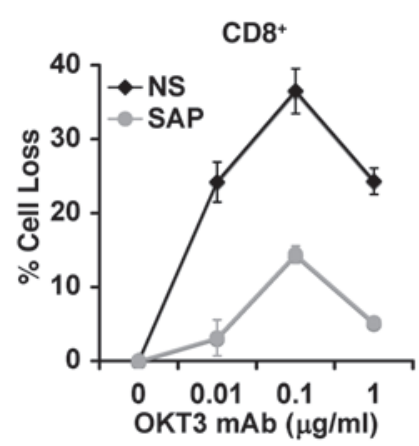

C
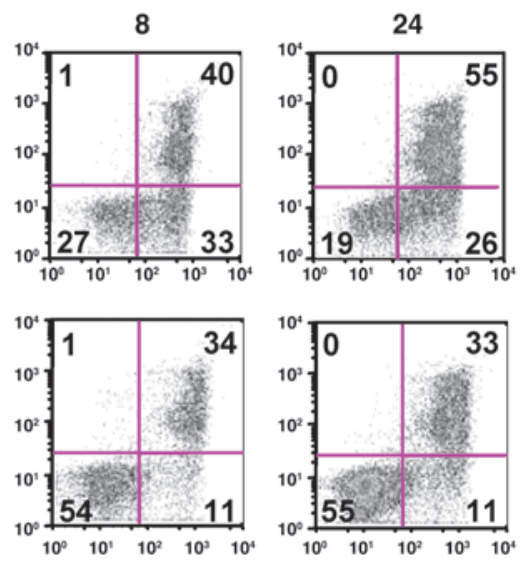
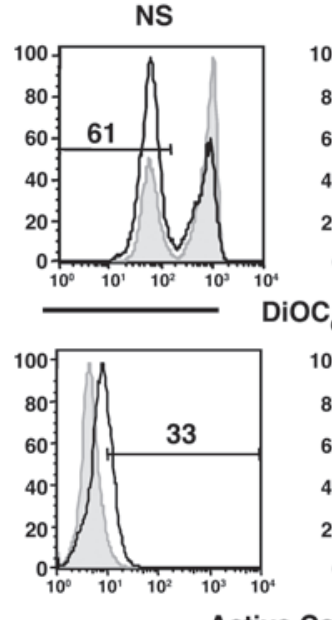

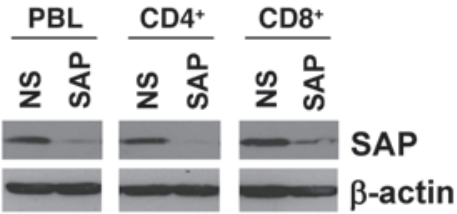

Active Casp 3

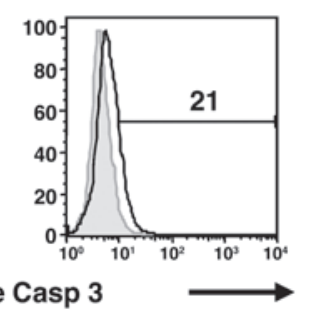

Figure 2

Silencing SAP expression in normal T cells results in defective RICD. (A) Left: Activated PBLs were transfected with SAP-specific siRNA or NS control siRNA and cultured in IL-2 for an additional 4 days, then restimulated with increasing doses of OKT3 mAb. Alternatively, CD4+ or CD8 ${ }^{+} \mathrm{T}$ cells were purified from PBLs prior to siRNA transfection. Cell death at 24 hours was assessed by PI staining and measured by flow cytometry. Right: Lysates prepared from siRNA-transfected cells were separated by SDS-PAGE and immunoblotted with SAP Ab to assess knockdown of SAP expression. $\beta$-Actin served as a loading control. (B) Activated PBLs were transfected with siRNA and cultured as described above. Cells were then restimulated with $200 \mathrm{ng} / \mathrm{ml}$ OKT3 mAb for 8 and 24 hours or left untreated $(0 \mathrm{hr})$, then stained with Annexin V-FITC and PI. The numbers in each quadrant indicate the percentage of cells in that quadrant. (C) siRNA-transfected PBLs were restimulated as described above for 24 hours or left untreated, then stained with $\mathrm{DiOC}_{6}$ or anti-caspase-3 Ab. Numbers indicate the percentage of cells with permeabilized mitochondria $\left(\mathrm{DiOC}_{6}{ }^{\circ}\right)$ or active caspase-3 after restimulation.

the control, although anti-CD3/CD28 stimulation was generally suboptimal for purified naive $\mathrm{T}$ cell subsets (Supplemental Figure $4 \mathrm{C}$ ). Moreover, there were no observed differences in the continued expansion and viability of activated XLP patient $\mathrm{T}$ cells relative to controls during prolonged culture with exogenous IL-2, whether from bulk PBLs or isolated T cell subsets (data not shown). These data are consistent with previous studies in which SAP-deficient $\mathrm{T}$ cells from both humans and mice exhibited normal or even increased responsiveness to primary T cell stimulation (14). Thus, we can define a consistent and marked deficit in sensitivity to RICD in XLP patient T cells that cannot be attributed to impaired activation or expansion in vitro.

SAP is necessary for optimal RICD. To address whether SAP is directly required for RICD, we transfected cycling, activated T cells from normal donors with SAP-specific or nonspecific (NS) control siRNA. Knockdown of SAP expression in activated PBLs (composed of mostly CD8 ${ }^{+} \mathrm{T}$ cells) conferred a striking resistance to TCRinduced apoptosis, as shown in Figure 2A. Protein expression was substantially reduced in SAP siRNA-transfected cells compared with NS controls (Figure 2A and Supplemental Figure 5A). This defect was also observed in purified $\mathrm{CD}^{+}$and more strikingly in $\mathrm{CD}^{+} \mathrm{T}$ cell subsets. Loss of SAP expression debilitated the deathinducing capacity of TCR signal transduction, as early markers of apoptosis were significantly reduced in $\mathrm{T}$ cells that received SAP siRNA (Figure 2, B and C). By contrast, SAP knockdown had little effect on activation or proliferation of naive T cells, as measured by CD69/CD25 induction, thymidine incorporation, and CFSE dilution (Supplemental Figure 5, B and C). These results imply that SAP is largely dispensable for initial $\mathrm{T}$ cell activation and proliferation but critical for optimal apoptosis of $\mathrm{T}$ cells triggered specifically by TCR restimulation. Hence, SAP deficiency is directly responsible for the loss of sensitivity to TCR-induced apoptosis observed in XLP patients. A similar trend was observed using EBV-specific $\mathrm{CD}^{+} \mathrm{T}$ cells transfected with NS or SAP siRNA and restimulated with irradiated, autologous $\mathrm{EBV}^{+}$lymphoblastoid cells (LCLs) (Supplemental Figure 6). Thus, loss of SAP can confer resistance to RICD in an antigen-specific manner, which may be directly relevant to deadly EBV-induced FIM noted in XLP patients. 
A

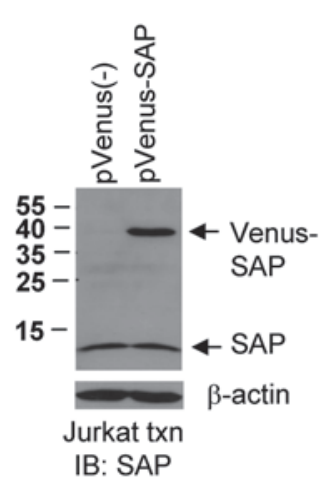

B
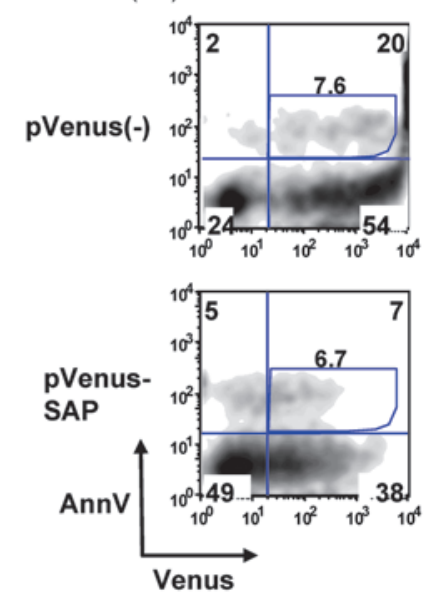

8
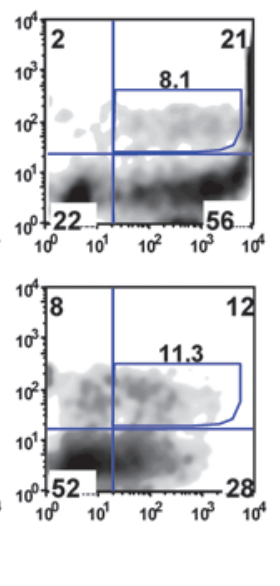

C

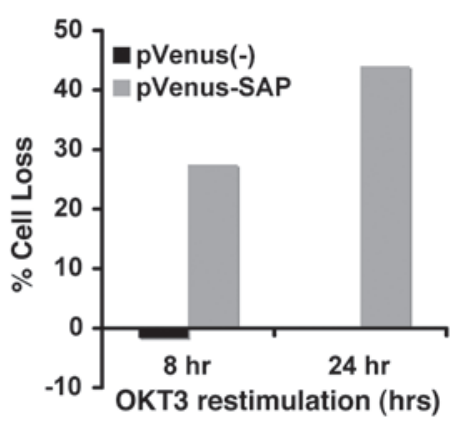

Figure 3

Ectopic expression of SAP restores RICD in XLP Pt4 T cells. (A) Jurkat cells were transfected with pVenus-SAP or pVenus alone and lysed after 48 hours. Lysates were separated by SDS-PAGE and immunoblotted with anti-SAP Ab. $\beta$-Actin served as a loading control. (B) Activated XLP Pt4 PBLs were transfected with pVenus-SAP or pVenus alone and incubated overnight. After removal of dead cells, cells were restimulated with $500 \mathrm{ng} / \mathrm{ml}$ OKT3 for 8-24 hours. Apoptosis was measured by Annexin V staining. The additional Annexin $\mathrm{V}^{+}$gate in the top right quadrants excluded Venus ${ }^{+}$cells that could not be compensated due to bright fluorescence. (C) Transfected cells were treated as described in $\mathbf{B}$. The percentage of cell loss was calculated based on viable cells present before and after restimulation.

We also asked whether ectopic expression of SAP in XLP T cells could restore RICD sensitivity. We constructed a fluorescent Venus-SAP fusion protein that was readily expressed when transfected in Jurkat T cells (Figure 3A). Expression of Venus-SAP in activated Pt4 PBLs increased RICD in transfected cells relative to Venus protein alone, as measured by increased Annexin $V$ staining (Figure 3B) and loss of viable cells after restimulation (Figure 3C). These data further implicate SAP as a critical mediator of RICD signal transduction.

FASL and BIM induction via TCR restimulation is impaired in the absence of SAP. To explore why TCR-induced apoptosis is largely blocked in SAP-deficient $\mathrm{T}$ cells, we conducted a comparative microarray analysis of gene expression 6 hours after TCR restimulation in normal donor $\mathrm{T}$ cells transfected with either NS or SAP-specific siRNA. RNA collected and amplified from siRNAtransfected $T$ cells was hybridized to spotted arrays against reference RNA pooled from activated $T$ cells gathered from 6 different donors. This experimental design limited differences in gene expression between siRNA-treated and reference samples to changes specifically associated with TCR re-engagement. We identified 357 genes with at least 2-fold greater expression in NS versus SAP-specific siRNA (Figure 4A), including many genes transcriptionally upregulated by TCR restimulation in effector $\mathrm{T}$ cells such as cytokines (e.g., IL-4 and IL-10), coreceptor ligands (e.g., programmed cell death 1 ligand 1 [PD-L1] and CTLA-4), and various apoptosis effector molecules. These results suggested a global downmodulation of TCR-induced target genes, consistent with a proximal signaling defect.

The list of differentially affected apoptosis-related genes included FASL (FASLG) and BIM (BCL2L11), encoding 2 molecules normally induced by TCR restimulation that directly participate in the execution of RICD. TCR-induced upregulation of FASL and BIM was substantially reduced in SAP knockdown cells as confirmed by real-time PCR (Figure 4B). Immunoblotting also showed less TCR-induced protein expression of full-length (FL) FASL and all
3 BIM protein isoforms (Figure 4C). FL FASL is cleaved by matrix metalloproteinases upon transport to the plasma membrane, shedding soluble FasL into the extracellular milieu while generating a $17-\mathrm{kDa}$-terminal fragment retained in the cytosol that was evident in our blots (Figure 4C) (25). Although FL FASL protein was slightly elevated in SAP-knockdown cells before restimulation, all forms of FASL decreased over time after TCR ligation. This suggested that while enzymatic processing of FASL could proceed, FL FASL protein was not replenished by TCR triggering when SAP expression was diminished.

We next authenticated these findings in XLP patient T cells. The normally robust induction of both FASL and BIM mRNA and protein was almost totally abrogated in restimulated T cells from XLP Pt4 (Figure 4, D and E). This effect was arguably more pronounced than the partial inhibition of gene upregulation noted in SAPknockdown cells, perhaps due to the incomplete blockade of SAP expression in cells transfected with siRNA (Figure 4C). Generation of soluble FASL in supernatants from restimulated SAP-knockdown cells and Pt4 T cells was also severely impaired compared with controls (Figure 4F), consistent with our immunoblotting results. These results were corroborated in several XLP patients that we studied and in purified $\mathrm{CD}^{+}$and $\mathrm{CD}^{+}$subsets from $\mathrm{Pt} 7$ (Supplemental Figure 7).

The substantial contribution of FASL and BIM to the execution of RICD is well established. However, our microarray analysis yielded many genes besides FASL and BIM that were poorly induced by TCR restimulation in the absence of SAP. We validated these data for several genes in both SAP-knockdown and XLP T cells (Supplemental Figure 8). In addition, knockdown of class I MHC-restricted T cell-associated molecule (CRTAM) or PD-L1 resulted in minor decreases in RICD sensitivity (Supplemental Figure 9). The relative contribution of additional SAP-dependent TCR-induced target genes for RICD remains to be tested, but it is likely that poor induction of FASL and BIM explains part of the RICD resistance noted in activated XLP T cells. 

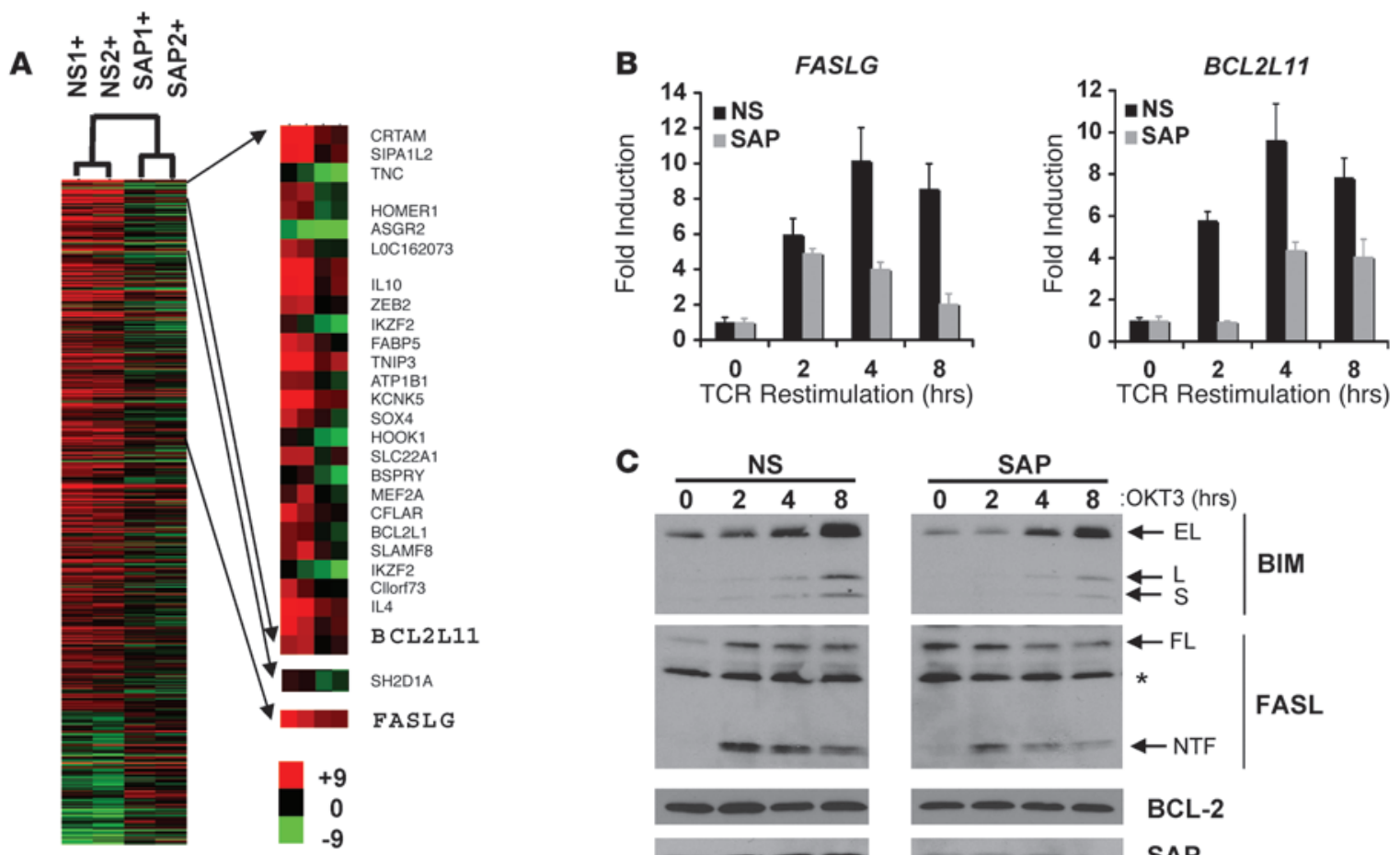

C
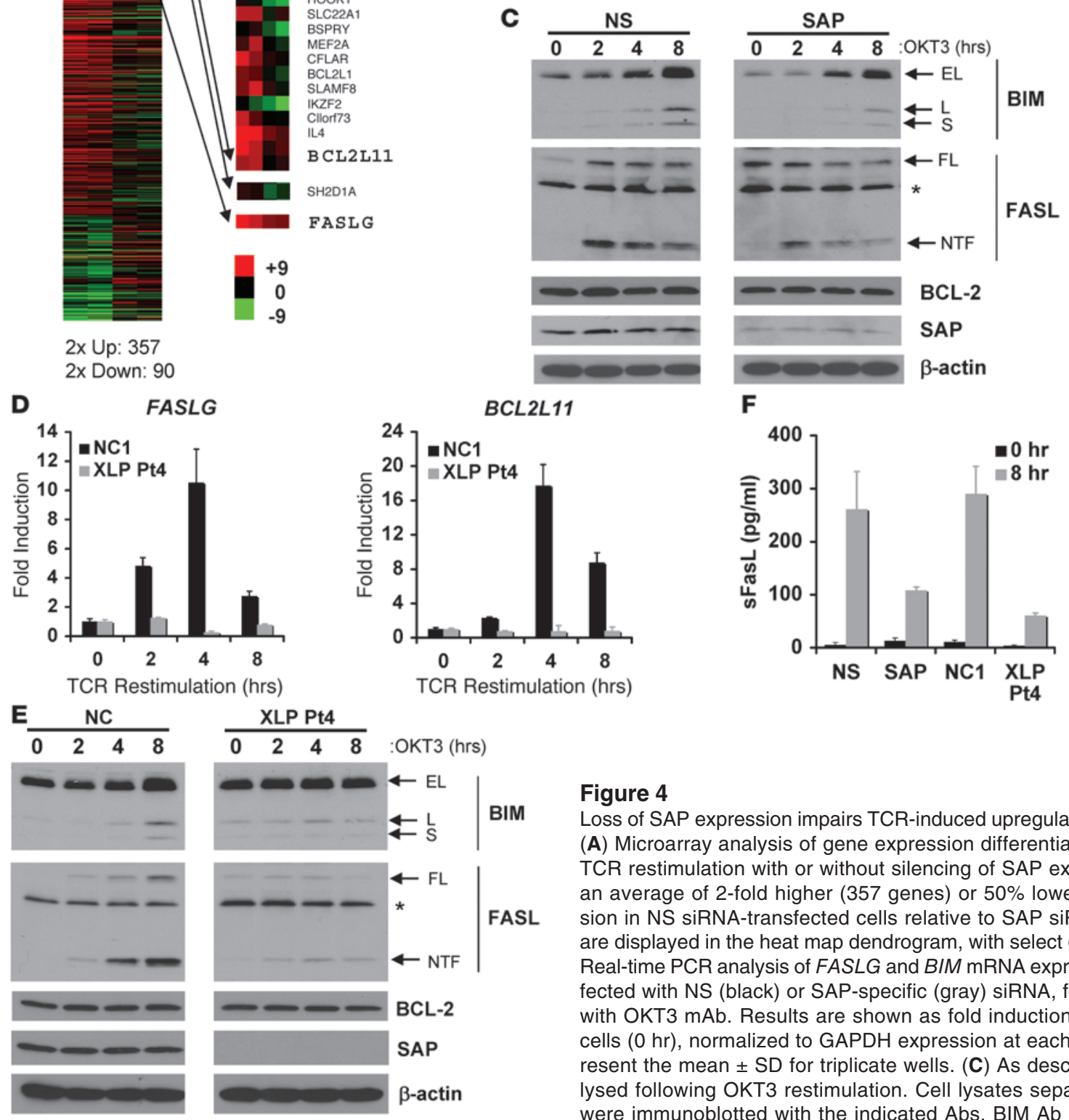

Figure 4

Loss of SAP expression impairs TCR-induced upregulation of FASL and BIM. (A) Microarray analysis of gene expression differentially affected by 6-hour TCR restimulation with or without silencing of SAP expression. Genes with an average of 2 -fold higher (357 genes) or $50 \%$ lower (90 genes) expression in NS siRNA-transfected cells relative to SAP siRNA-transfected cells are displayed in the heat map dendrogram, with select genes highlighted. (B) Real-time PCR analysis of FASLG and BIM mRNA expression in T cells transfected with NS (black) or SAP-specific (gray) siRNA, following restimulation with OKT3 mAb. Results are shown as fold induction relative to untreated cells ( $0 \mathrm{hr}$ ), normalized to GAPDH expression at each time point. Data represent the mean \pm SD for triplicate wells. (C) As described in B, cells were lysed following OKT3 restimulation. Cell lysates separated by SDS-PAGE were immunoblotted with the indicated Abs. BIM Ab detected all 3 protein isoforms (extra-long [EL], long [L], and short [S]); FasL Ab detected FL and a cytosolic N-terminal fragment (NTF). (D) Real-time PCR analysis of FASLG and $B I M$ mRNA expression in activated T cells from a normal donor (black) and XLP Pt4 (gray) following restimulation as described in B. (E) As described in $D$, cells were lysed following OKT3 restimulation. Cell lysates separated by SDS-PAGE were immunoblotted with the indicated Abs. (F) ELISA detection of soluble FasL (sFasL) in supernatants collected from cells restimulated as described above for 0 or 8 hours. 
A

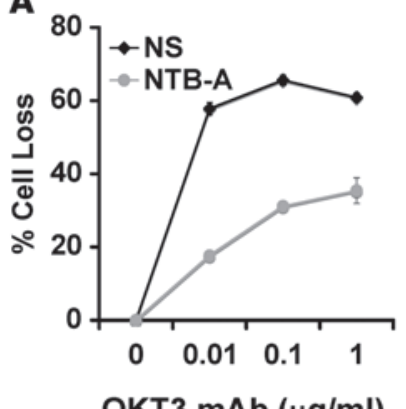

OKT3 mAb $(\mu \mathrm{g} / \mathrm{ml})$

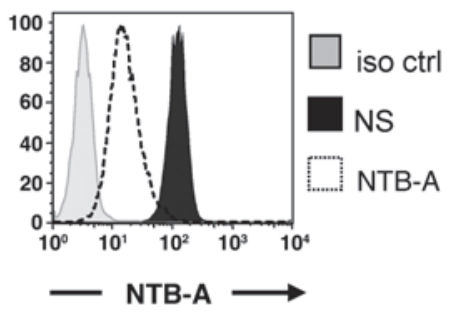

D

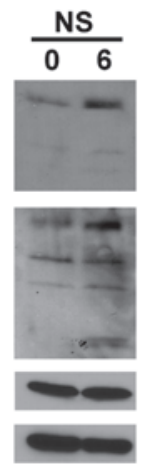

B OKT3 $(\mathrm{hr})$ :

0

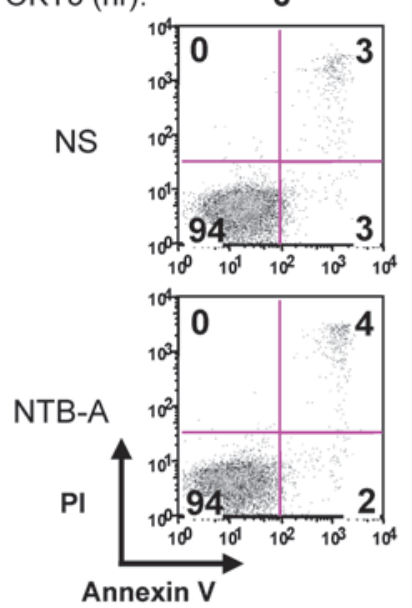

NS
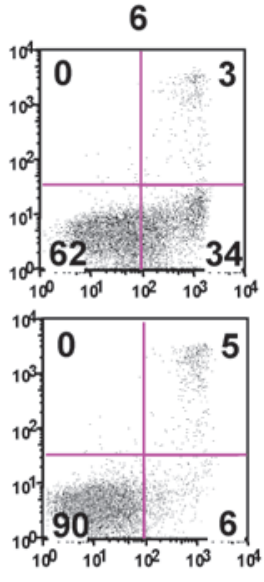

C

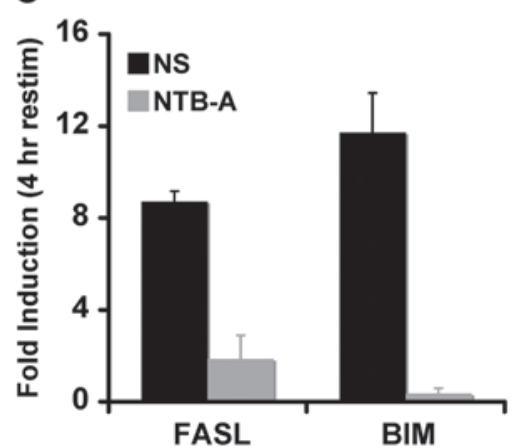

$\frac{\text { NTB-A }}{0.6}$

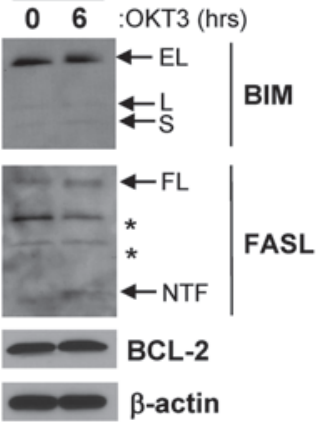

\section{E}

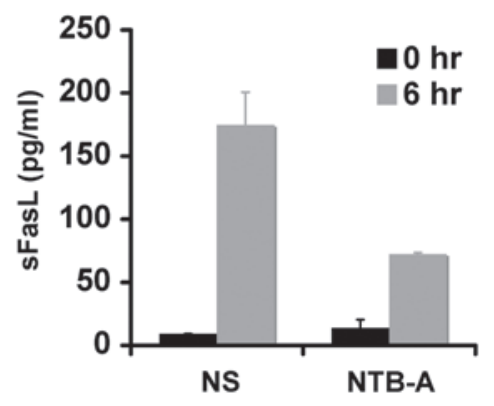

Figure 5

Silencing NTB-A expression reduces TCR-induced upregulation of FASL and BIM and RICD. (A) Activated PBLs transfected with NTB-A-specific siRNA or NS control siRNA were restimulated with increasing doses of OKT3 mAb, and apoptosis was assessed at 24 hours by PI staining. Surface expression of NTB-A was measured by flow cytometry for NS and NTB-A siRNA-transfected cells relative to cells stained with isotype control Ab. (B) PBLs transfected as described in A were restimulated with OKT3 mAb for 0 or 6 hours, then stained with Annexin V-FITC and PI. Numbers in each quadrant indicate the percentage of cells in that quadrant. (C) Real-time PCR analysis of FASLG and BIM mRNA expression in T cells transfected with NS or NTB-A-specific siRNA, following restimulation with OKT3 mAb. Results are shown as fold induction relative to untreated cells $(0 \mathrm{hr}$ ), normalized to GAPDH expression. (D) As described in C, cells were lysed following OKT3 restimulation. Cell lysates separated by SDS-PAGE were immunoblotted with the indicated Abs. (E) ELISA detection of SFasL in supernatants collected from siRNA-transfected cells restimulated with OKT3 for 0 or 6 hours.

The SLAM family receptor NTB-A contributes to RICD. The SLAM family of immunomodulatory receptors utilizes SAP as an $\mathrm{SH} 2$ adaptor protein for recruiting kinases (e.g., FYN) that can amplify signaling cascades initiated by the TCR or NK activating receptors to affect cytokine production, cytotoxicity, and related functions (14). We therefore asked which SLAM receptor(s) might be required for driving SAP-dependent RICD. SLAM receptor expression was assessed on activated $\mathrm{T}$ cells by flow cytometry, and RNAi-based silencing was used to test for differences in apoptosis sensitivity following TCR restimulation. Surface expression of SLAMF6, also known as NTB-A, far exceeded that of the other SLAM family members, including SLAM, LY9, 2B4, CD84, and CD2-like receptor activating cytotoxic cells (CRACC), on activated T cells (Figure 5A and Supplemental Figure 10A). Despite an inability to silence NTB-A completely, we found that only knockdown of NTB-A afforded marked protection against TCR-induced apoptosis (Figure 5, A and B, and Supplemental Figure 10B). Thus, a decrease only in NTB-A expression recapitulated the apoptotic defect conferred by SAP deficiency. By contrast, reducing expression of other SLAM receptors actually appeared to increase death sensitivity in some cases (Supplemental Figure 10). Hence, the balance of SLAM receptor expression may help to determine cell fate following TCR restimulation.

We next asked whether decreasing expression of NTB-A also resulted in impaired restimulation-induced upregulation of FASL and BIM, as we had observed in SAP-deficient T cells. RT-PCR, immunoblotting, and soluble FASL ELISA detection illuminated a substantially reduced induction of both molecules in cells transfected with NTB-A siRNA relative to NS controls (Figure 5, C-E). With the exception of ICAM-1, several SAP-dependent TCRinduced target genes identified in our microarray analysis were also poorly induced in NTB-A-silenced T cells, including CRTAM and PD-L1 (Supplemental Figure 11). These data strongly suggest that SAP influences TCR signaling events largely through NTB-A, including upregulation of effector molecules required for RICD.

To test whether SAP physically associates with NTB-A in restimulated $\mathrm{T}$ cells, we immunoprecipitated SAP from $\mathrm{T}$ cell lysates before and after brief TCR restimulation, followed by sensitive mass spectrometry analysis of associated proteins. We detected SLAM and 
A

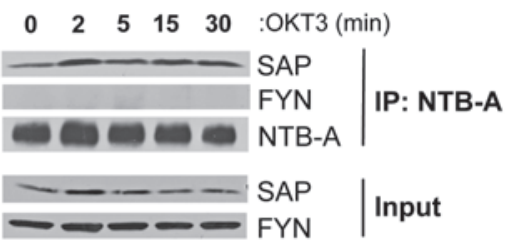

C

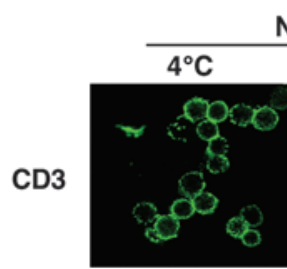

NC
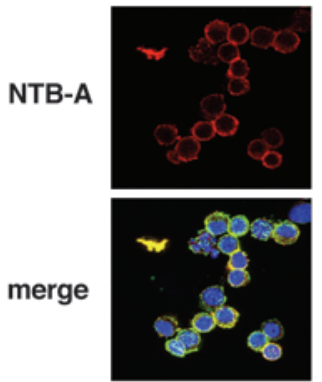

D

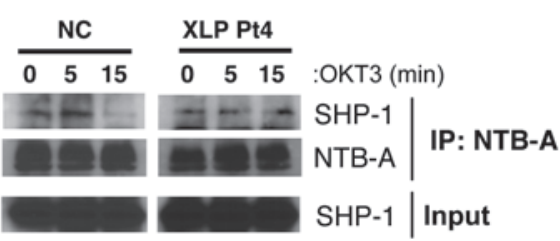

F
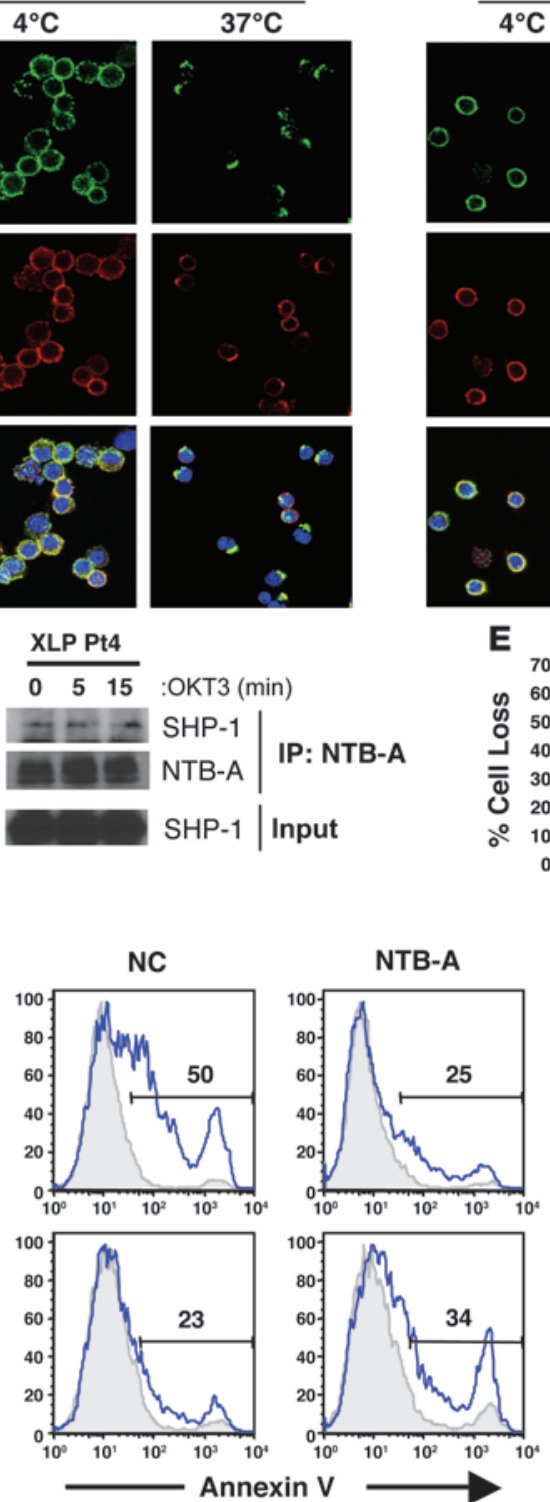

B
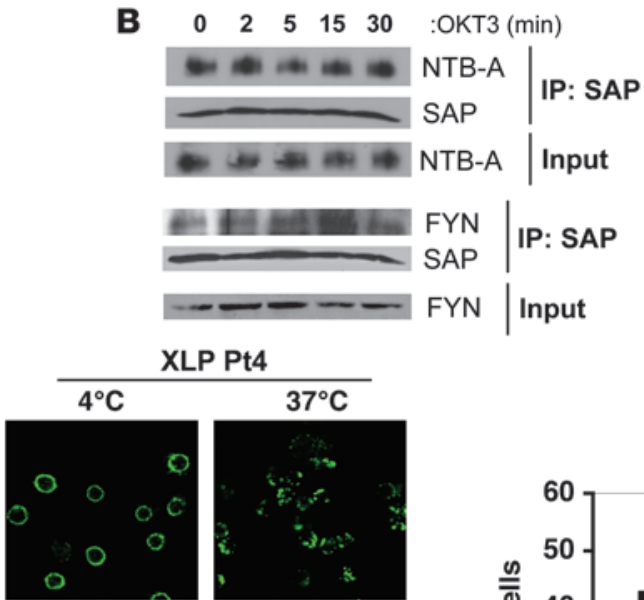

$37^{\circ} \mathrm{C}$
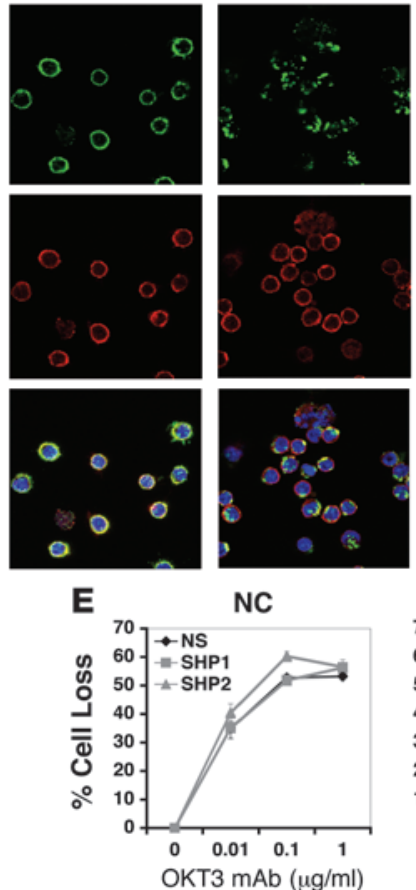

OKT3 mAb $(\mu \mathrm{g} / \mathrm{ml})$
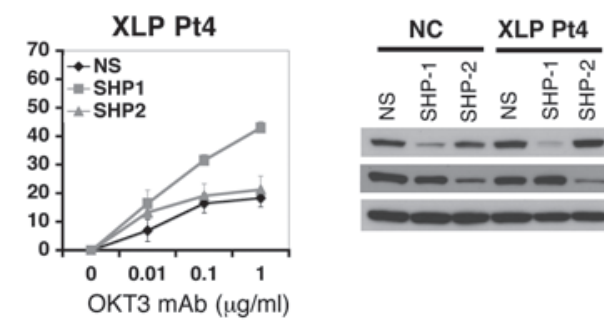

- - - SHP-1 $--\infty-$ SHP-2 $\beta$-actin

G
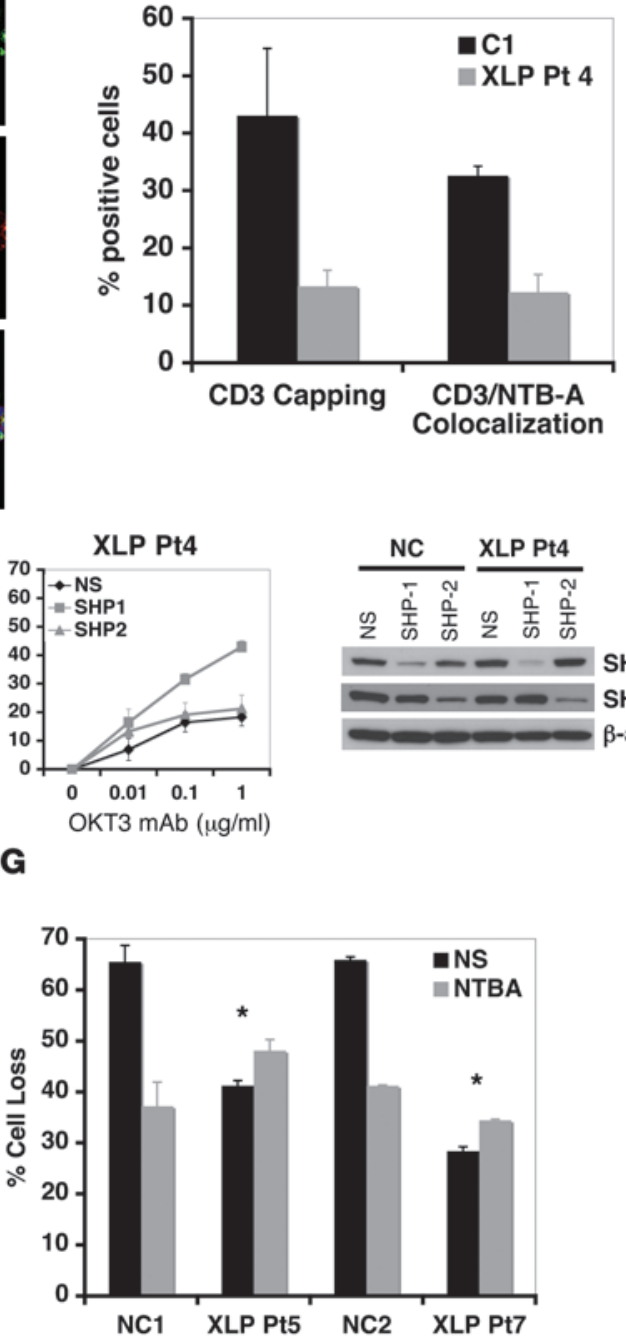

Figure 6

NTB-A influences TCR-induced apoptosis signals through competitive association with SAP and SHP-1. (A) NTB-A was immunoprecipitated from PBL lysates after a short time course of OKT3 restimulation. Immunoprecipitates were separated by SDS-PAGE and immunoblotted for the presence of SAP and NTB-A. SAP expression in input lysates is shown at bottom for comparison. (B) SAP was immunoprecipitated from PBL lysates and immunoblotted as described in A. (C) Cells were stained with mAbs detecting CD3 (OKT3, green) and NTB-A (red) and left on ice ( $\left.4^{\circ} \mathrm{C}\right)$ or warmed to $37^{\circ} \mathrm{C}$ for 30 minutes. Left: Cells were imaged by confocal microscopy, with nuclei counterstained in blue. Right: Quantitative analysis based on counting more than 200 cells from multiple fields. (D) NTB-A was immunoprecipitated from lysates prepared from normal donor or XLP Pt4 PBLs following OKT3 stimulation. Immunoprecipitates were separated by SDS-PAGE and immunoblotted for the presence of SHP-1 and NTB-A. (E) Normal donor or XLP Pt4 PBLs were transfected with NS, SHP-1-specific, or SHP-2-specific siRNA and restimulated with increasing doses of OKT3 mAb. Apoptosis was measured by PI staining. Knockdown efficiency was assessed by immunoblotting. (F and $\mathbf{G})$ Normal donor or XLP PBLs were transfected with NS or NTB-A siRNA and restimulated with $100 \mathrm{ng} / \mathrm{ml}$ OKT3 mAb. Apoptosis was measured by Annexin V (F) or PI staining (G). ${ }^{*} P<0.04$. 
A

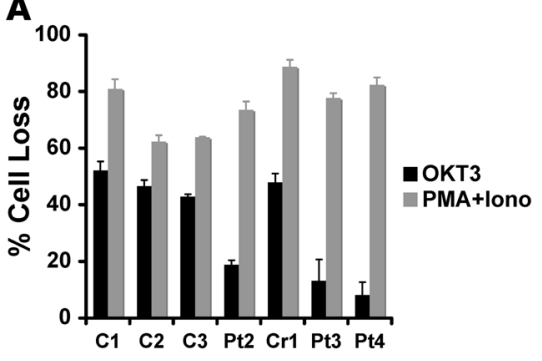

D

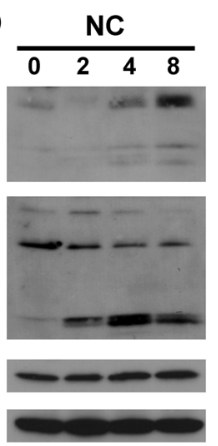

B

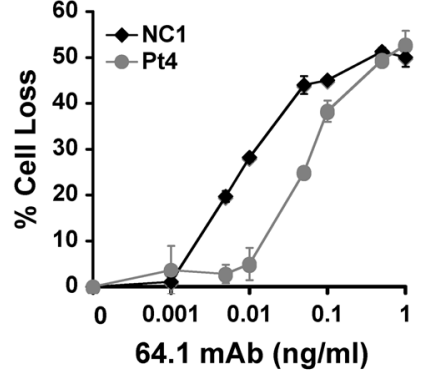

$\mathbf{E}$

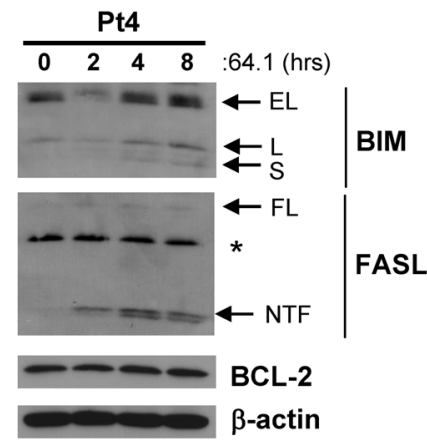

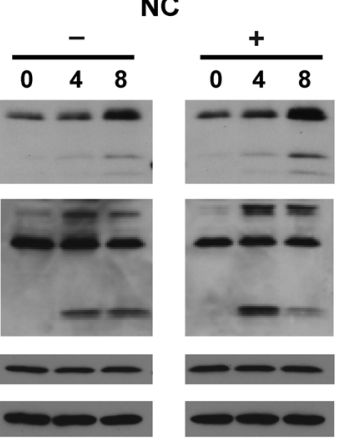

C

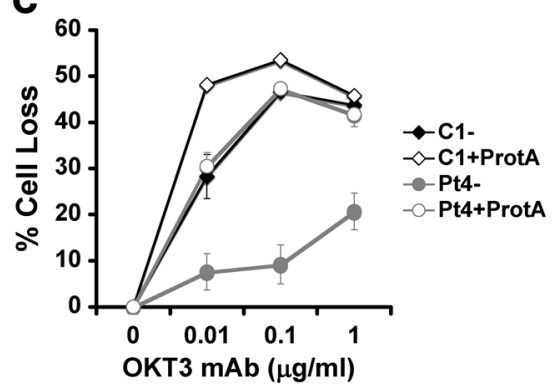

Pt4

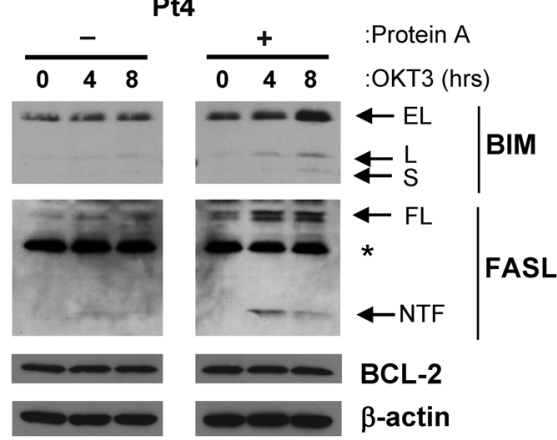

Figure 7

An increase in TCR signal strength bypasses the requirement for SAP in restimulation-induced T cell death. (A) Activated T cells derived from normal donors or XLP patients were restimulated with $100 \mathrm{ng} / \mathrm{ml} \mathrm{OKT3}$ or $50 \mathrm{ng} / \mathrm{ml}$ PMA plus $1 \mu \mathrm{g} / \mathrm{ml}$ ionomycin. Apoptosis was measured 24 hours after treatment by PI staining and flow cytometry. (B) T cells from a normal control or XLP Pt4 were treated with increasing doses of the anti-CD3 mAb 64.1, and apoptosis was determined after 24 hours as described above. (C) As described in B, PBLs were treated with increasing doses of OKT3 mAb in the absence or presence of $1 \mathrm{mg} / \mathrm{ml}$ Protein A, and apoptosis was measured after 24 hours. (D) As described in B, PBLs were restimulated with $64.1 \mathrm{mAb}$ and lysed. Lysates separated by SDS-PAGE were immunoblotted with the indicated Abs. (E) As described in B, PBLs were restimulated with $200 \mathrm{ng} / \mathrm{ml}$ OKT3 mAb with or without $1 \mathrm{mg} / \mathrm{ml}$ Protein A. Cells were lysed, separated by SDS-PAGE, and immunoblotted with the indicated Abs.

NTB-A coprecipitated with SAP using this approach, regardless of whether cells were restimulated (data not shown). However, we observed that SAP association with immunoprecipitated NTB-A increased after TCR restimulation, suggesting TCR signaling indirectly enhances this interaction (Figure 6A). When total SAP was immunoprecipitated over a time course of restimulation, the amount of bound NTB-A appeared to decrease slightly at 5 minutes, before increasing at 15-30 minutes (Figure 6B). This may be explained by initial recruitment of some SAP to SLAM receptors other than NTB-A, or to other cellular compartments. As a negative control, CD84 failed to immunoprecipitate with SAP (Supplemental Figure 12), suggesting that SAP only selectively associates with certain SLAM family receptors in activated T cells. Increased SAP recruitment to NTB-A upon TCR restimulation is congruent with naive $\mathrm{T}$ cell activation, for which NTB-A can have costimulatory function (26). However, we failed to detect FYN in NTB-A immunoprecipitates (Figure 6A), although some FYN could be detected in association with SAP (Figure 6B). These data suggest that FYN does not participate specifically in NTB-A signaling in restimulated $\mathrm{T}$ cells. Consistent with this hypothesis, knockdown of FYN did not significantly alter RICD sensitivity (Supplemental Figure 13A). Moreover, ectopic expression of a Venus-SAP mutant fusion protein incapable of binding FYN (R78A) restored RICD sensitivity to a similar extent as WT SAP (Supplemental Figure 13B). Taken together, these data indicate that the potentiation of RICD by SAP and NTB-A is FYN independent.
SLAM has previously been shown to localize with TCR clusters following stimulation of naive human T cells (27). We found a proportion of NTB-A colocalized with clusters of CD3 after TCR re-engagement (Figure 6C), placing NTB-A and SAP in close proximity to the active TCR signaling complex. In contrast, we observed less NTB-A colocalization with CD3 clusters in XLP patient cells after restimulation (Figure 6C and Supplemental Figure 14). Moreover, CD3 aggregation appeared more disordered in XLP patient cells (Figure 6C), consistent with diminished TCR signaling reflected in impaired target gene upregulation. These data suggest that NTB-A and SAP have an architectural role in organizing CD3 clusters, which may serve to augment TCR signal strength.

SAP is also known to compete with $\mathrm{SH} 2$ domain-containing protein tyrosine phosphatases SHP-1 and SHP-2 for binding to conserved tyrosine-based motifs in the cytoplasmic tails of SLAM receptors $(13,28)$. In NK cells, constitutive association of NTB-A with SHP-1 is lost upon pervanadate-simulated activation, concomitant with increased recruitment of SAP (28). We observed a similar phenomenon in activated T cells; less SHP-1 was complexed with NTB-A 15 minutes after restimulation (Figure 6D), consistent with increased recruitment of SAP to NTB-A thereafter. However, SHP-1 levels did not decrease in NTB-A immunoprecipitates derived from restimulated XLP Pt $4 \mathrm{~T}$ cells (Figure 6D), suggesting that SHP-1 remains associated with NTB-A when SAP is not present to compete for binding. 


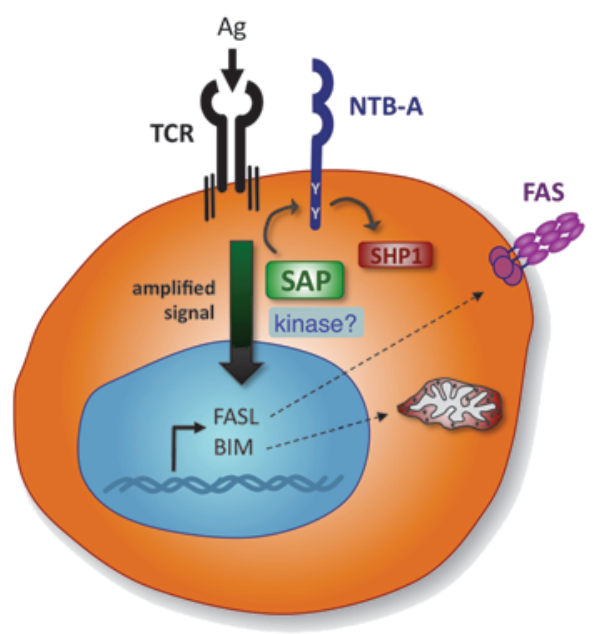

\section{Figure 8}

Model for the involvement of SAP/NTB-A signaling in augmenting TCR signal strength for apoptosis. Left: In previously activated T lymphocytes, TCR restimulation triggers increased association of NTB-A with SAP and colocalization with TCR-CD3 complexes, dislodging SHP-1 in the process. Exclusion of this phosphatase and potential recruitment of additional kinases ensures that optimal TCR signal transduction proceeds for the induction of downstream target genes such as FASL and BIM, which contribute to apoptosis execution. Right: In this fashion, SAP potentiates TCR signal strength past the required threshold for apoptosis induction in normal $T$ cells. In SAP-deficient T cells, overall TCR signal strength is downmodulated below this threshold, allowing cells to escape deletion.
Both SHP-1 and SHP-2 can dephosphorylate a wide variety of substrates, serving to downmodulate multiple signaling cascades in hematopoietic cells. To test whether SHP-1 or SHP-2 actively influenced the outcome of TCR restimulation in XLP Pt4 T cells, we measured RICD following knockdown of either molecule. Reduction of SHP-1, but not SHP-2, restored RICD sensitivity in XLP Pt4 T cells, particularly at high doses of anti-CD3 (Figure 6E). This effect was validated using normal donor cells with siRNA knockdown of SAP, as simultaneous knockdown of SHP-1 surmounted the apoptosis resistance caused by SAP silencing (Supplemental Figure 15). Knockdown of SHP-1 alone had little effect on RICD sensitivity in normal donor T cells (Figure 6E and Supplemental Figure 15).

Given the sustained association of NTB-A with SHP-1 in XLP $T$ cells, we then asked whether silencing NTB-A expression could also reverse RICD resistance in XLP T cells. In contrast to the protective effect observed in normal controls, knockdown of NTB-A in XLP patient $\mathrm{T}$ cells resulted in a small but statistically significant increase in RICD sensitivity (Figure 6, F and G). This result implies that NTB-A can transmit inhibitory signals upon restimulation of XLP T cells, similar to its function in dampening the cytotoxic potential of SAP-deficient NK cells (28). Taken together, these findings indicate that the counterposing functions of SAP and SHP-1, coordinated through NTB-A, can influence apoptosis sensitivity following TCR restimulation of activated T cells.

The necessity of SAP and NTB-A for TCR-induced death can be bypassed by increasing TCR signal strength. General downmodulation of many TCR-inducible target genes suggested that SAP-NTB-A complexes contribute importantly to the efficiency of TCR signaling in preactivated $T$ cells. Consistent with this concept, stimulation with phorbol ester (PMA) and ionomycin, reagents that bypass early TCR signaling events, triggered equivalent apoptosis in control and XLP patient T cells (Figure 7A). This result implied that SAP plays a role in proximal TCR signaling, whereas the biochemical machinery downstream of this point was capable of driving apoptosis in both normal and SAP-deficient cells.

The initial descriptions of apoptosis following $\mathrm{T}$ cell restimulation indicated that apoptosis is achieved when a certain threshold of TCR signal strength is applied, based partly on the qualitative avidity of the recognized antigen-MHC complex $(29,30)$. It has been reported that some consequences of TCR signal transduction, such as NF- $\mathrm{kB}$ p50 nuclear translocation, are reduced in SAPknockout murine $\mathrm{T}$ cells (31). Therefore, we hypothesized that
SAP-NTB-A signaling may potentiate TCR signal strength to help reach the threshold required for death. To test this possibility, we employed a different anti-CD3 mAb known as 64.1 to crosslink the TCR in normal and XLP Pt4 T cells. For naive T cell stimulation, 64.1 is a stronger mAb than OKT3, as it can trigger more robust responses such as a larger $\mathrm{Ca}^{++}$flux and more proliferation (32). At low doses of $64.1 \mathrm{mAb}$, presumably below saturation of surface TCR complexes, XLP patient T cells remained resistant to RICD. However, higher doses of $64.1 \mathrm{mAb}$ affected similar RICD sensitivity in normal control and XLP Pt4 T cells (Figure 7B and Supplemental Figure 16A), demonstrating that increasing the strength of restimulation bypasses the need for SAP and overcomes the influence of SHP-1 in executing RICD. Similarly, apoptosis was also reestablished in XLP Pt4 T cells treated with the weaker OKT3 mAb if it was hyper-cross-linked with high-capacity $\mathrm{Fc}$ binding protein $\mathrm{A}$ (Figure 7C). Consistent with triggering RICD, these stronger treatment regimens partially restored the induction of FASL and BIM in XLP T cells as well as the associated caspase activation (Figure 7, D and E, and Supplemental Figure 16, B-D). The same rescue effect was noted in other XLP patients and applied to other genes (e.g., CRTAM and PD-L1) that were poorly induced in SAP-deficient cells without protein A treatment (Supplemental Figure 17). These data further implicate SAP in augmenting TCR signal strength up to the threshold required for RICD of activated T cells, and suggests that defective RICD in XLP patients may be overcome by treatment approaches that artificially boost or bypass proximal TCR signals.

\section{Discussion}

The mechanistic connection between SAP deficiency and excessive lymphoproliferation, particularly in the context of infectious challenge, is a fundamental unanswered question of this severe disease. Here we demonstrate that despite being dispensable for primary stimulation, SAP is critical for ensuring the elimination of activated human T cells following TCR restimulation. Our results also provide what is, to our knowledge, the first insight into the immunological role of NTB-A in T cell regulation.

We propose a model by which SAP plays a key role in setting the threshold for RICD, particularly with respect to $\mathrm{CD}^{+} \mathrm{T}$ cells (Figure 8). TCR re-engagement triggers SAP recruitment to immunoreceptor tyrosine-based switch motifs found on the cytoplasmic tail of NTB-A, which can dislodge SHP-1 from the receptor based on its binding properties (33). We also found that NTB-A is 
closely complexed with the TCR upon restimulation on activated $\mathrm{T}$ cells, an event not previously observed for this SLAM family receptor. SAP association with NTB-A nullified SHP-1 inhibition, and TCR signaling proceeded to turn on key target genes, such as FASL and BIM, that trigger programmed cell death. This leads to a robust homeostatic apoptosis of $\mathrm{T}$ cells in restimulation conditions, which would be expected to prevail during a persistent viral infection. SAP association with other SLAM family members may occur upon restimulation, although our RNAi screen data suggest that only NTB-A plays a major role in influencing RICD. Moreover, our data suggest that SAP-dependent recruitment of FYN to NTB-A is not required for RICD, so it remains to be determined whether other kinases act to amplify the apoptosis-promoting signal conveyed by SAP-NTB-A association. For example, SAP can interact with LCK, which has been shown to phosphorylate SLAM and CD84 $(27,34)$. Our data provide a coherent molecular model for the severe pathogenic antigen-induced lymphoproliferation observed in children with this genetic defect.

Enhanced clonotypic expansion of SAP $-/-\mathrm{CD}^{+} \mathrm{T}$ cells, concomitant with reduced apoptosis following primary stimulation, was recently described in OT-I TCR transgenic mice and connected to poor upregulation of the p53 homolog p73 in OVA-stimulated naive $\mathrm{CD}^{+} \mathrm{T}$ cells $(21)$. In that study, death was assessed within 48 hours after a single antigen challenge and by definition did not measure RICD of activated effector cells upon rechallenge with antigen. Moreover, the relative contribution of $\mathrm{p} 73$ to $\mathrm{T}$ cell apoptosis may be restricted to death immediately following primary stimulation, as p73-deficient $T$ cells show no defect in restimulation-induced apoptosis $(35,36)$. However, although we also observed less apoptosis following primary stimulation of XLP T cells, this trend did not correlate with decreased $\mathrm{p} 73$ induction relative to controls (Supplemental Figure 18). Moreover, we failed to detect any significant induction of p73 in restimulated human $\mathrm{T}$ cells, or any change in apoptosis sensitivity after p73 siRNA transfection (data not shown). Rather, our results suggest that SAP acts as an amplifier of proximal signals triggered by TCR re-engagement that is important for RICD of both $\mathrm{CD}^{+}$and $\mathrm{CD}^{+} \mathrm{T}$ cells, ensuring optimal induction of downstream target genes including the pro-apoptotic executioner molecules FASL and BIM.

Regulation of lymphocyte homeostasis by apoptosis adds to the expanding list of immunomodulatory functions attributed to SAP and SLAM family receptors such as NTB-A. However, apoptosis dysregulation in XLP is distinct from the apoptosis defect in autoimmune lymphoproliferative syndrome (ALPS) in several interesting respects. SAP has no bearing on the inherent susceptibility of an activated $\mathrm{T}$ cell to extrinsic or intrinsic apoptosis signals; direct crosslinking of Fas or removal of IL-2 effectively kills XLP patient T cells. Hence, cytokine withdrawal-mediated $\mathrm{T}$ cell contraction theoretically proceeds normally in XLP patients once antigen is cleared and may be accelerated according to our data (Figure 1B). In addition to well-established defects in humoral immunity, this explains why XLP patients do not develop chronic lymphadenopathy, splenomegaly, or autoimmune manifestations characteristic of ALPS (37). However, the paradoxic propensity for B cell lymphomagenesis found in both XLP and ALPS may derive from defective FAS-mediated killing of B cells by activated T cells bearing FASL.

In cloning SAP as the affected gene in XLP, Sayos et al. also noted that SAP could block the recruitment of SHP-2 to SLAM (13). SAP may serve a similar purpose in augmenting TCR- induced signals by displacing SHP-1 from NTB-A. We observed that NTB-A could colocalize with clustered TCR-CD3 complexes following restimulation, consistent with a function in modulating RICD. This is biochemically analogous to NTB-A signaling in NK cells, which contributes to the cytotoxic potential of NK cells against EBV-infected targets along with 2B4 (28). SAP also acts as an adaptor protein to recruit SH3 domain-containing kinases such as FYN, LCK, and NCK1 to SLAM family receptors, which can enhance the cascade of protein phosphorylation that accompanies TCR signaling (38-40). Our data imply that SAP can potentiate RICD independently of FYN, but more work is required to dissect the specific biochemistry connecting SAPNTB-A interactions to downstream TCR signal transduction. Whether homotypic NTB-A interactions occur in cis or trans on activated $\mathrm{T}$ cells also remains to be determined in our system. Furthermore, our RNAi screen of the SLAM family suggests that a balance of SLAM receptor expression may control RICD sensitivity, perhaps through differential association with SAP, SHP-1, and/or other binding partners. Although anti-CD3 Ab-induced death of activated $\mathrm{T}$ cells has been observed at the single cell level, which would ostensibly preclude a role for NTB-A as we described, physiological antigen stimulation of $\mathrm{T}$ cells is invariably a cell-cell transaction, making it possible that SLAM receptor interactions with antigen-presenting cells or adjacent $\mathrm{T}$ cells directly influence signaling for RICD. Nevertheless, the global suppression of many TCR-induced target genes in the absence of SAP and NTB-A implies that these molecules directly affect proximal TCR signaling events.

Our observation that the requirement for SAP can be overridden by artificially increasing the TCR signal strength supports the threshold model of RICD. It is generally accepted that a quantitatively and qualitatively strong restimulation signal is required to surpass the threshold necessary for triggering apoptosis. We propose that the SAP-NTB-A interaction plays an integral role in reaching the required threshold. In the absence of SAP, TCR signaling effectively becomes weaker, resembling a partial agonist signal that involves negative feedback loops like those controlled by SHP-1 in response to weakly binding ligands (41).

Despite extensive work in vitro, the full importance of RICD in vivo has remained unknown (24). XLP may now prove illustrative of the significance of this self-regulatory mechanism. Defective RICD likely contributes to several clinical features of the disease, notably T cell-driven LPD and HLH. Without SAP, unbridled effector $T$ cell expansion secondary to viral infection or other antigenic stimuli, especially in antigen-rich environments such as the lungs, proceeds without counterbalancing propriocidal death. Indeed, we noted lung nodules with T cell infiltrates in XLP Pt2, Pt3, and Pt8, anecdotally linked to VZV vaccination and detection of HHV6 in lung tissue or blood, respectively. In turn, this overly expanded $\mathrm{T}$ cell compartment can drive activation and proliferation of other hematopoietic cell subsets. Hence, the apoptotic defect that we have discovered provides a unifying mechanism for the diverse pathological features of XLP, including pulmonary/CNS vasculitis, pulmonary nodules, and EBV-associated FIM.

We believe this scenario is particularly relevant to the unique susceptibility of XLP patients to EBV infection for several reasons. First, EBV normally induces a vigorous cellular immune response, greatly expanding the pool of EBV-specific effector $\mathrm{T}$ cells. Second, recent evidence from mice indicates that $\mathrm{T}$ cell/ $\mathrm{B}$ cell interactions are inherently weaker in the absence of SAP 
(42). This implies that any encounter between an EBV-specific XLP T cell and an EBV-infected B cell is short lived, constituting an attenuated restimulation signal, likely below the threshold required for RICD. Third, SAP-deficient EBV-specific T cells fail to kill EBV-infected B cells due to impaired 2B4 and NTB-Amediated cytotoxicity, with the consequence that the source of abundant EBV antigen is never effectively cleared. The defect in FASL upregulation we uncovered in SAP-deficient $\mathrm{T}$ cells may contribute to impaired cytotoxic potential in this setting. These elements culminate in the "perfect storm" of EBV-associated FIM/HLH, stemming from unchecked proliferation of EBV-specific $\mathrm{T}$ cells (especially $\mathrm{CD} 8^{+} \mathrm{T}$ cells) that fail to undergo RICD in response to repeated antigenic stimulation.

Without curative allogeneic stem cell transplantation, the prognosis for XLP patients remains poor, as only $30 \%$ of XLP patients survive past age 10, and the mortality rate for EBV-associated FIM/HLH is approximately $96 \%$ (8). Our findings can now refocus efforts toward novel therapeutic approaches for XLP and related disorders based on this clear and specific apoptosis defect. Removal of the potential source of EBV-associated antigens via pre-emptive B cell depletion, or administration of SHP-1 inhibitors to generally boost TCR signal strength, could prevent or retard HLH progression by constraining numbers of responding $\mathrm{T}$ cells. Collectively, our demonstration of impaired RICD in human SAP-deficient $\mathrm{T}$ cells provides mechanistic insight on XLP-associated LPD, underscores the physiological importance of this autoregulatory cell death mechanism in a unique disease setting, and reiterates the fact that XLP manifestations are not dependent on the presence of EBV infection. These observations may stimulate new approaches to the clinical management of XLP and other disorders of lymphocyte apoptosis and homeostasis.

\section{Methods}

Cells and treatments. Patients were enrolled and blood samples were obtained with informed consent under protocols approved by the Institutional Review Boards of Cincinnati Children's Hospital and the NIH. PBMCs were isolated from whole blood by Ficoll-Hypaque density gradient centrifugation, washed, and resuspended at $10^{6}$ cells $/ \mathrm{ml}$ in complete RPMI 1640 medium (Lonza) containing 10\% FCS, $2 \mathrm{mM}$ glutamine, and $100 \mathrm{U} / \mathrm{ml}$ each of penicillin and streptomycin (Invitrogen). T cells were activated with $1 \mu \mathrm{g} / \mathrm{ml}$ each of anti-CD3 $\varepsilon$ and anti-CD28 mAbs (BD Biosciences), or $1 \mu \mathrm{g} / \mathrm{ml} \mathrm{OKT3}$ $\mathrm{mAb}$ (anti-CD3e) plus $25 \mathrm{U} / \mathrm{ml}$ rhIL-2 (Peprotech). In some experiments, mitogenic $\mathrm{T}$ cell activation was tested with $5 \mu \mathrm{g} / \mathrm{ml}$ concanavalin A or $1 \mu \mathrm{g} / \mathrm{ml}$ phytohemagglutinin. After 3 days, activated T cells were thoroughly washed and then cultured in complete RPMI-1640 medium supplemented with $100 \mathrm{U} / \mathrm{ml}$ rhIL-2 (Roche Applied Science) for at least 7 days before apoptosis assays were conducted. Activated $\mathrm{T}$ cell subsets were separated using CD4 or CD8 Microbeads and MACS magnetic bead cell separation (Miltenyi Biotec), with purities of greater than $95 \%$ and $90 \%$, respectively.

Apoptosis assays and flow cytometry. To test sensitivity to apoptotic stimuli, activated T cells $\left(10^{5}\right.$ cells/well) were plated in triplicate in 96-well roundbottomed plates and treated with the following reagents: agonistic anti-Fas mAb APO1.3 (2-200 ng/ml; Alexis) plus $200 \mathrm{ng} / \mathrm{ml}$ Protein A, anti-CD3ع $\mathrm{mAb}$ OKT3 $(10-1,000 \mathrm{ng} / \mathrm{ml})$ with or without $1 \mu \mathrm{g}$ Protein A, anti-CD3ع $\mathrm{mAb} 64.1$ (1-1,000 ng/ml; gift of E.M. Shevach, National Institute of Allergy and Infectious Diseases, NIH), or staurosporine ( $2 \mu \mathrm{M}$ in DMSO; SigmaAldrich). For UV-induced apoptosis, cells were exposed to $40,000 \mathrm{~mJ} / \mathrm{cm}^{2}$ UV irradiation using a Stratalinker UV crosslinker (Stratagene). At 24 hours after treatment, cells were stained with $1 \mu \mathrm{g} / \mathrm{ml}$ propidium iodide and collected for 15 seconds per sample on a FACScan flow cytometer (BD Bio- sciences). Cell death was quantified as percentage cell loss $=(1-$ number of viable cells (treated) / number of viable cells (untreated)]) $\times 100$. Alternatively, cells were stained with Annexin V-FITC for 10 minutes at room temperature and measured by flow cytometry. Mitochondrial permeability

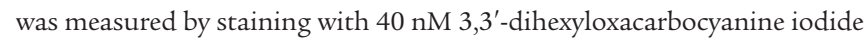
(DiOC6) (EMD Biosciences) for 15 minutes at $37^{\circ} \mathrm{C}$ before flow cytometry analysis. Active caspase- 3 was measured by intracellular flow cytometry using a PE-conjugated Ab (BD Biosciences - Pharmingen). Active caspase- 8 was assessed using a colorimetric assay kit for enzymatic activity (BioVision). For surface staining, cells were stained with $5 \mu \mathrm{g}$ of fluorochrome-conjugated Abs against CD2, CD4, CD8, CD25, CD69, CD95, SLAM, 2B4, CD84, NTB-A, Ly9, CRACC, and CRTAM (BD Biosciences). Detection of intracellular SAP was performed as previously described using the rat anti-SH2D1A mAb KST-3, with comparison of results to a previously obtained reference range (43).

For EBV-specific kill assays, EBV-specific $\mathrm{CD}^{+} \mathrm{T}$ cells were generated as previously described using autologous, irradiated EBV-transformed LCLs as antigen-presenting cells (44). After 7-14 days in culture with IL-2, EBV T cells were restimulated with varying ratios of irradiated LCL with or without $1 \mu \mathrm{g} / \mathrm{ml}$ anti-class I MHC blocking Ab (clone W6/32; Santa Cruz Biotechnology Inc.).

RNAi. Activated human PBLs were prepared as described above and transfected with 200 pmol of siRNA oligonucleotides specific for the target protein (Stealth Select siRNA; Invitrogen) or a NS control oligo (Invitrogen) using the Amaxa Nucleofection system (Lonza; Program T-020). Knockdown of SAP expression was confirmed by immunoblotting, as outlined above. All subsequent assays were performed 4 days after transfection at the point of maximal knockdown.

pVenus-SAP transfection. WT or R78A SAP was PCR amplified and cloned into pMyc-Venus via EcoRI and NotI restriction sites to create Venus-SAP fusion protein constructs. Transfection tests were performed in Jurkat E6.1 cells by electroporation of $10 \mu \mathrm{g} / 0.4 \times 10^{6}$ cells using the BTX electroporation system. For activated PBLs, $8 \times 10^{6}$ cells/sample were nucleofected with $10 \mu \mathrm{g}$ DNA using Amaxa (Program T-023). At 18 hours after transfection, viable cells were isolated using a dead cell removal kit (Miltenyi Biotec) and restimulated with $500 \mathrm{ng} / \mathrm{ml} \mathrm{OKT3}$ for 8-24 hours.

Mircoarray and real-time PCR analysis. RNA was isolated from 2 normal donor $T$ cells transfected with NS or SAP siRNA following 0 or 6 hours after OKT3 restimulation using Trizol (Invitrogen) and RNeasy mini-columns (Qiagen). For spotted arrays, purified RNA was amplified using the Ovation Aminoallyl Amplification System (NuGEN), labeled with Cy5 using the Cy5 Reactive Dye Pack (GE Healthcare), and cleaned using Vivaspin columns (VivaScience AG). Amplified RNA ( $2 \mu \mathrm{g}$ ) was hybridized to Hsbb 23K human spotted arrays (National Institute of Allergy and Infectious Diseases Mircoarray Research Facility) versus Cy3-labeled reference RNA pooled from 6 normal donor cycling T cells. Data were analyzed using GenePix and mAdb software. For real-time PCR analysis, cDNA was prepared from $1 \mu \mathrm{g}$ RNA using the Superscript III RT Kit (Invitrogen). EXPRESS SYBR Green qPCR Supermix (Invitrogen) was used for subsequent PCR with specific primers for each target gene, analyzed on an ABI PRISM 7900HT Sequence Detection System (Applied Biosystems).

Immunoblotting and immunoprecipitations. Activated $\mathrm{T}$ cells were lysed in 1\% NP-40 lysis buffer (50 mM Tris pH 7.4, $150 \mathrm{mM} \mathrm{NaCl}, 1 \mathrm{mM}$ EDTA, $1 \%$ NP- $40,0.5 \%$ sodium deoxycholate) with Complete protease inhibitors (Roche) for 15 minutes on ice, then cleared by centrifugation. Protein concentration was determined by BCA assay (Pierce Biotechnology), and $40 \mu \mathrm{g}$ total protein was separated by SDS-PAGE. After transfer to nitrocellulose, blots were probed with the following Abs: anti-SAP, antiFynT (Cell Signal Technology), anti-FasL (Ab3; EMD Biosciences), antiBIM (Stressgen), anti-BCL-xL, anti-BCL-2 (BD Biosciences - Pharmin- 
gen), anti-NTB-A (Novus), anti-SHP-1, anti-SHP-2, anti-CD3ל (Santa Cruz Biotechnology Inc.), and anti- $\beta$-actin mAb (clone AC-15; SigmaAldrich). Bound Abs were detected using the appropriate HRP-conjugated secondary Abs (Southern Biotech) and ECL (Pierce Biotechnology). For IP, $2-5 \times 10^{7}$ cells were lysed as described above following stimulation, then incubated overnight with 5-10 $\mu$ g SAP or NTB-A (BioLegend) Abs plus protein G-conjugated agarose beads (Sigma-Aldrich). Bead precipitates were washed 4 times in lysis buffer prior to SDS-PAGE analysis. For mass spectrometry analysis, SAP IP from $10^{8}$ cells were separated by SDS-PAGE and stained with colloidal Coomassie blue dye. Bands were excised, digested, and subjected to mass spectrometry analysis as previously described (45).

Confocal microscopy. Activated $\mathrm{T}$ cells were stained for 20 minutes on ice with $10 \mu \mathrm{g} / \mathrm{ml} \mathrm{OKT3} \mathrm{mAb}$ and $5 \mu \mathrm{g} / \mathrm{ml}$ Alexa Fluor 488-conjugated anti-mouse IgG (Invitrogen), then washed with PBS. Stained cells were left on ice $\left(4^{\circ} \mathrm{C}\right)$ or warmed to $37^{\circ} \mathrm{C}$ to allow for restimulation, then fixed in $4 \%$ paraformaldehyde and cytospun on glass slides $(145 \mathrm{~g}$ for 5 minutes). Cells were blocked for 30 minutes in PBS plus 10\% FCS, then incubated with $1 \mu \mathrm{g} / \mathrm{ml}$ anti-NTB-A (BioLegend), washed, and stained with Alexa Fluor 594-conjugated anti-mouse IgG1 (30 minutes per incubation). Following 5 minutes of incubation in Hoechst 33342 to stain nuclei, coverslips were mounted using Fluoromount-G (Southern Biotech). Fluorescent images were acquired on a Leica SP5 confocal microscope using a $\times 63$ oil immersion objective. For per-cell quantitation of NTB-A/CD3 colocalization, images were deconvolved using Huygens Essential software, then analyzed using Imaris 6.2.1 software. Colocalization of green signal with red signal was calculated for each image according to the automatic threshold, using a standard red signal intensity threshold value of 29.41 .

Mutational analysis of SH2D1A. Genomic DNA was extracted from peripheral blood according to standard protocols. The coding region and the exon-intron boundaries of the SH2D1A gene were amplified by PCR using primers flanking each of the 4 exons. Direct sequencing of PCR products was performed using the $\mathrm{ABI} 3730 \mathrm{XL}$ sequencer (PE Biosystems) with the same primers used for PCR amplification.

Statistics. Data in apoptosis assays represent mean \pm SD for triplicate wells, representative of several independent experiments. Where noted, statistical significance was determined in apoptosis assays using a Student's $t$ test $(P<0.05)$. Pearson correlation coefficients were determined for molecular colocalization in confocal microscopy images using Imaris 6.2.1 software $(0<P<1)$.

\section{Acknowledgments}

We thank the patients and their families, as well as Lewis Silverman at Dana Farber Cancer Institute for providing blood samples on XLP Pt5. We also thank Juraj Kabat for help with confocal microscopy analysis and Pamela Schwartzberg for critical reading of the manuscript. We acknowledge the clinical laboratory testing performed by the Cincinnati Children's Hospital Diagnostic Immunology Laboratory as well as care for the patients provided by the bone marrow transplant physicians, nurses, and staff at Cincinnati Children's Hospital. This research was supported by the Intramural Research Program of the NIH, National Institute of Allergy and Infectious Diseases. A.L. Snow was supported by a Pharmacology Research Associate Training Program Fellowship from the National Institute of General Medical Sciences.

Received for publication April 12, 2009, and accepted in revised form July 22, 2009.

Address correspondence to: Michael J. Lenardo, Building 10, Rm 11N311, 9000 Rockville Pike, Bethesda, Maryland 20892, USA. Phone: (301) 496-6754; Fax: (301) 480-7352; E-mail: lenardo@nih.gov.
1. Lenardo, M., et al. 1999. Mature T lymphocyte apoptosis--immune regulation in a dynamic and unpredictable antigenic environment. Annu. Rev. Immunol. 17:221-253.

2. Brenner, D., Krammer, P.H., and Arnold, R. 2008. Concepts of activated T cell death. Crit. Rev. Oncol. Hematol. 66:52-64.

3. Snow, A.L., et al. 2008. Critical role for BIM in T cell receptor restimulation-induced death. Biol. Direct. 3:34.

4. Bidere, N., Su, H.C., and Lenardo, M.J. 2006 Genetic disorders of programmed cell death in the immune system. Annu. Rev. Immunol. 24:321-352.

5. Oliveira, J.B., et al. 2007. NRAS mutation causes a human autoimmune lymphoproliferative syndrome. Proc. Natl. Acad. Sci. U. S. A. 104:8953-8958.

6. Purtilo, D.T., Cassel, C.K., Yang, J.P., and Harper, R. 1975. X-linked recessive progressive combined variable immunodeficiency (Duncan's disease). Lancet. 1:935-940.

7. Purtilo, D.T., and Grierson, H.L. 1991. Methods of detection of new families with X-linked lymphoproliferative disease. Cancer Genet. Cytogenet. 51:143-153.

8. Seemayer, T.A., et al. 1995. X-linked lymphoproliferative disease: twenty-five years after the discovery. Pediatr. Res. 38:471-478.

9. Gaspar, H.B., Sharifi, R., Gilmour, K.C., and Thrasher, A.J. 2002. X-linked lymphoproliferative disease: clinical, diagnostic and molecular perspective. Br. J. Haematol. 119:585-595.

10. Nichols, K.E., Ma, C.S., Cannons, J.L., Schwartzberg, P.L., and Tangye, S.G. 2005. Molecular and cellular pathogenesis of X-linked lymphoproliferative disease. Immunol. Rev. 203:180-199.
11. Coffey, A.J., et al. 1998. Host response to EBV infection in X-linked lymphoproliferative disease results from mutations in an SH2-domain encoding gene. Nat. Genet. 20:129-135.

12. Nichols, K.E., et al. 1998. Inactivating mutations in an SH2 domain-encoding gene in X-linked lymphoproliferative syndrome. Proc. Natl. Acad. Sci.U.S. A. 95:13765-13770.

13. Sayos, J., et al. 1998. The X-linked lymphoproliferative-disease gene product SAP regulates signals induced through the co-receptor SLAM. Nature. 395:462-469.

14. Ma, C.S., Nichols, K.E., and Tangye, S.G. 2007. Regulation of cellular and humoral immune responses by the SLAM and SAP families of molecules. Annu. Rev. Immunol. 25:337-379.

15. Bassiri, H., Janice Yeo, W.C., Rothman, J., Koretzky, G.A., and Nichols, K.E. 2008. X-linked lymphoproliferative disease (XLP): a model of impaired antiviral, anti-tumor and humoral immune responses. Immunol. Res. 42:145-159.

16. Chen, G., et al. 2005. Signaling lymphocyte activation molecule-associated protein is a negative regulator of the $\mathrm{CD} 8 \mathrm{~T}$ cell response in mice. J. Immunol. 175:2212-2218.

17. Czar, M.J., et al. 2001. Altered lymphocyte responses and cytokine production in mice deficient in the X-linked lymphoproliferative disease gene SH2D1A/DSHP/SAP. Proc. Natl. Acad. Sci. U. S. A. 98:7449-7454

18. Wu, C., et al. 2001. SAP controls T cell responses to virus and terminal differentiation of $\mathrm{TH} 2$ cells. Nat. Immunol. 2:410-414.

19. Yin, L., et al. 2003. Mice deficient in the X-linked lymphoproliferative disease gene sap exhibit increased susceptibility to murine gammaherpesvirus-68 and hypo-gammaglobulinemia. J. Med. Virol. 71:446-455.

20. Crotty, S., McCausland, M.M., Aubert, R.D., Wherry, E.J., and Ahmed, R. 2006. Hypogammaglobulinemia and exacerbated CD8 T-cell-mediated immunopathology in SAP-deficient mice with chronic LCMV infection mimics human XLP disease. Blood. 108:3085-3093.

21. Chen, G., et al. 2007. Increased proliferation of CD8 $+\mathrm{T}$ cells in SAP-deficient mice is associated with impaired activation-induced cell death. Eur. J. Immunol. 37:663-674.

22. Strasser, A., Jost, P.J., and Nagata, S. 2009. The many roles of FAS receptor signaling in the immune system. Immunity. 30:180-192.

23. Ramaswamy, M., et al. 2007. Cutting edge: Rac GTPases sensitize activated T cells to die via Fas. J. Immunol. 179:6384-6388.

24. Lenardo, M.J. 1991. Interleukin-2 programs mouse alpha beta T lymphocytes for apoptosis. Nature. 353:858-861.

25. Schulte, M., et al. 2007. ADAM10 regulates FasL cell surface expression and modulates FasL-induced cytotoxicity and activation-induced cell death. Cell Death Differ. 14:1040-1049.

26. Valdez, P.A., et al. 2004. NTB-A, a new activating receptor in T cells that regulates autoimmune disease. J. Biol. Chem. 279:18662-18669.

27. Howie, D., et al. 2002. Molecular dissection of the signaling and costimulatory functions of CD150 (SLAM): CD150/SAP binding and CD150-mediated costimulation. Blood. 99:957-965.

28. Bottino, C., et al. 2001. NTB-A [correction of GNTB-A], a novel SH2D1A-associated surface 
molecule contributing to the inability of natural killer cells to kill Epstein-Barr virus-infected B cells in X-linked lymphoproliferative disease. J. Exp. Med. 194:235-246.

29. Combadiere, B., et al. 1996. Qualitative and quantitative contributions of the T cell receptor zeta chain to mature T cell apoptosis. J. Exp. Med. 183:2109-2117.

30. Combadiere, B., Reis e Sousa, C., Germain, R.N., and Lenardo, M.J. 1998. Selective induction of apoptosis in mature $\mathrm{T}$ lymphocytes by variant $\mathrm{T}$ cell receptor ligands. J. Exp. Med. 187:349-355.

31. Cannons, J.L., et al. 2004. SAP regulates T(H)2 differentiation and PKC-theta-mediated activation of NF-kappaB1. Immunity. 21:693-706.

32. Wacholtz, M.C., and Lipsky, P.E. 1993. Anti-CD3stimulated $\mathrm{Ca} 2+$ signal in individual human peripheral T cells. Activation correlates with a sustained increase in intracellular $\mathrm{Ca} 2+1$. J. Immunol. 150:5338-5349.

33. Hwang, P.M., et al. 2002. A "three-pronged" binding mechanism for the SAP/SH2D1A SH2 domain: structural basis and relevance to the XLP syndrome. EMBO J. 21:314-323.
34. Tangye, S.G., Nichols, K.E., Hare, N.J., and van de Weerdt, B.C. 2003. Functional requirements for interactions between CD84 and Src homology 2 domain-containing proteins and their contribution to human $\mathrm{T}$ cell activation. J. Immunol. 171:2485-2495.

35. Lissy, N.A., Davis, P.K., Irwin, M., Kaelin, W.G., and Dowdy, S.F. 2000. A common E2F-1 and p73 pathway mediates cell death induced by TCR activation. Nature. 407:642-645.

36. Senoo, M., Manis, J.P., Alt, F.W., and McKeon, F. 2004. p63 and p73 are not required for the development and p53-dependent apoptosis of T cells. Cancer Cell. 6:85-89.

37. Oliveira, J.B., and Fleisher, T. 2004. Autoimmune lymphoproliferative syndrome. Curr. Opin. Allergy Clin. Immunol. 4:497-503.

38. Li, C., Schibli, D., and Li, S.S. 2009. The XLP syndrome protein SAP interacts with $\mathrm{SH} 3$ proteins to regulate $\mathrm{T}$ cell signaling and proliferation. Cell Signal. 21:111-119.

39. Veillette, A. 2006. Immune regulation by SLAM family receptors and SAP-related adaptors. Nat. Rev. Immunol. 6:56-66.
40. Zhong, M.C., and Veillette, A. 2008. Control of $\mathrm{T}$ lymphocyte signaling by Ly108, a signaling lymphocytic activation molecule family receptor implicated in autoimmunity. J. Biol. Chem. 283:19255-19264.

41. Stefanova, I., et al. 2003. TCR ligand discrimination is enforced by competing ERK positive and SHP-1 negative feedback pathways. Nat. Immunol. 4:248-254.

42. Qi, H., Cannons, J.L., Klauschen, F., Schwartzberg, P.L., and Germain, R.N. 2008. SAP-controlled T-B cell interactions underlie germinal centre formation. Nature. 455:764-769.

43. Tabata, Y., et al. 2005. Rapid detection of intracellular SH2D1A protein in cytotoxic lymphocytes from patients with X-linked lymphoproliferative disease and their family members. Blood. 105:3066-3071.

44. Sharifi, R., et al. 2004. SAP mediates specific cytotoxic T-cell functions in X-linked lymphoproliferative disease. Blood. 103:3821-3827.

45. Wan, F., et al. 2007. Ribosomal protein S3: a KH domain subunit in NF-kappaB complexes that mediates selective gene regulation. Cell. 131:927-939. 